\title{
Hot horizontal branch stars in NGC 288 - effects of diffusion and stratification on their atmospheric parameters ${ }^{\star, \star \star, \star \star \star \star}$
}

\author{
S. Moehler ${ }^{1,2}$, S. Dreizler ${ }^{3}$, F. LeBlanc ${ }^{4}$, V. Khalack ${ }^{4}$, G. Michaud ${ }^{5}$, J. Richer ${ }^{5}$, A. V. Sweigart ${ }^{6}$, and F. Grundahl ${ }^{7}$ \\ 1 European Southern Observatory, Karl-Schwarzschild-Str. 2, 85748 Garching, Germany \\ e-mail: smoehler@eso.org \\ ${ }^{2}$ Institut für Theoretische Physik und Astrophysik, Olshausenstraße 40, 24118 Kiel, Germany \\ 3 Georg-August-Universität, Institut für Astrophysik, Friedrich-Hund-Platz 1, 37077 Göttingen, Germany \\ e-mail: dreizler@astro.physik.uni-goettingen.de \\ 4 Département de Physique et d'Astronomie, Université de Moncton, Moncton, New Brunswick, E1A 3E9, Canada \\ e-mail: [francis.leblanc;viktor.khalak] @umoncton.ca \\ 5 Département de physique, Université de Montréal, Montréal, Québec, H3C 3J7, Canada \\ e-mail: [michaudg; jacques.richer]@umontreal.ca \\ ${ }^{6}$ NASA Goddard Space Flight Center, Exploration of the Universe Division, Code 667, Greenbelt MD 20771, USA \\ e-mail: allen. sweigart@gmail.com \\ 7 Stellar Astrophysics Centre, Department of Physics \& Astronomy, University of Århus, Ny Munkegade 120, 8000 Århus C, \\ Denmark \\ e-mail: fgj@phys.au.dk
}

Received 31 October 2013 / Accepted 11 March 2014

\section{ABSTRACT}

\begin{abstract}
Context. NGC 288 is a globular cluster with a well-developed blue horizontal branch (HB) covering the $u$-jump that indicates the onset of diffusion. It is therefore well suited to study the effects of diffusion in blue HB stars.

Aims. We compare observed abundances with predictions from stellar evolution models calculated with diffusion and from stratified atmospheric models. We verify the effect of using stratified model spectra to derive atmospheric parameters. In addition, we investigate the nature of the overluminous blue HB stars around the $u$-jump.

Methods. We defined a new photometric index $s z$ from uvby measurements that is gravity-sensitive between $8000 \mathrm{~K}$ and $12000 \mathrm{~K}$. Using medium-resolution spectra and Strömgren photometry, we determined atmospheric parameters $\left(T_{\text {eff }}, \log g\right)$ and abundances for the blue HB stars. We used both homogeneous and stratified model spectra for our spectroscopic analyses.

Results. The atmospheric parameters and masses of the hot HB stars in NGC 288 show a behaviour seen also in other clusters for temperatures between $9000 \mathrm{~K}$ and $14000 \mathrm{~K}$. Outside this temperature range, however, they instead follow the results found for such stars in $\omega$ Cen. The abundances derived from our observations are for most elements (except $\mathrm{He}$ and $\mathrm{P}$ ) within the abundance range expected from evolutionary models that include the effects of atomic diffusion and assume a surface mixed mass of $10^{-7} M_{\odot}$. The abundances predicted by stratified model atmospheres are generally significantly more extreme than observed, except for $\mathrm{Mg}$. When effective temperatures, surface gravities, and masses are determined with stratified model spectra, the hotter stars agree better with canonical evolutionary predictions.

Conclusions. Our results show definite promise towards solving the long-standing problem of surface gravity and mass discrepancies for hot HB stars, but much work is still needed to arrive at a self-consistent solution.
\end{abstract}

Key words. stars: horizontal-branch - stars: atmospheres - techniques: spectroscopic - globular clusters: individual: NGC 288

\section{Introduction}

Low-mass stars that burn helium in a core of about $0.5 M_{\odot}$ and hydrogen in a shell populate a roughly horizontal region in the optical colour-magnitude diagrams of globular clusters, which has earned them the name horizontal branch (HB) stars (ten Bruggencate 1927). The hot (or blue) HB stars near an effective temperature of $11500 \mathrm{~K}$ are of special interest because

^ Based on observations with the ESO Very Large Telescope at Paranal Observatory, Chile (proposal ID 71.D-0131).

$\star \star$ Tables 1 and 2 are available in electronic form at http://www. aanda.org

$\star \star \star$ The observed abundances plotted in Fig. 8 are only available at the CDS via anonymous ftp to cdsarc.u-strasbg. fr (130.79.128.5) or via

http://cdsarc.u-strasbg.fr/viz-bin/qcat?J/A+A/565/A100 they exhibit a number of intriguing phenomena associated with the onset of diffusion.

A large photometric survey of many globular clusters by Grundahl et al. (1999) demonstrated that the Strömgren $u$-brightness of blue HB stars suddenly increases near $11500 \mathrm{~K}$. This $u$-jump is attributed to a sudden increase in the atmospheric metallicity of the blue HB stars to super-solar values that is caused by the radiative levitation of heavy elements. A similar effect can be seen in broad-band $U, U-V$ photometric data (Ferraro et al. 1998, G1). Behr et al. $(1999,2000 a)$ and Moehler et al. (2000) confirmed with direct spectroscopic evidence that the atmospheric metallicity does indeed increase to solar or super-solar values for HB stars hotter than the $u$-jump. A list of earlier observations of this effect can be found in Moehler (2001). Later studies include Behr (2003, M3, M 13, M 15, M 68, M92, and NGC 288), Fabbian et al. (2005, NGC 1904), and Pace et al. (2006, NGC 2808). These findings also helped to 
understand the cause of the low-gravity problem: Crocker et al. (1988) and Moehler et al. (1995, 1997) found that hot HB stars when analysed with model spectra of the same metallicity as their parent globular cluster - show significantly lower surface gravities than expected from evolutionary tracks. Analysing them instead with more appropriate metal-rich model spectra reduces the discrepancies considerably (Moehler et al. 2000). The more realistic stratified model atmospheres of Hui-Bon-Hoa et al. (2000) and LeBlanc et al. (2009) reduce the discrepancies in surface gravity even more (see below for more details). Along with the enhancement of heavy metals, a decrease in the helium abundance by mass $Y$ is observed for stars hotter than $\approx 11500 \mathrm{~K}$, while cooler stars have normal helium abundances within the observational errors. The helium abundance for these hotter stars is typically between 1 and 2 dex smaller than the solar value (e.g. Behr 2003). A trend of the helium abundance relative to $T_{\text {eff }}$ was discussed by Moni Bidin et al. $(2009,2012)$. Finally, blue HB stars near $\approx 11500 \mathrm{~K}$ show a sudden drop in their rotation rates (Peterson et al. 1995; Behr et al. 2000b; Behr 2003), and in some globular clusters (e.g., M 13) a gap in their HB distribution.

The possibility of radiative levitation of heavy elements and gravitational settling of helium in HB stars had been predicted long ago by Michaud et al. (1983), but the discovery of its very sudden onset near $11500 \mathrm{~K}$ was a complete surprise. Quievy et al. (2009) have shown that helium settling in HB stars cooler than $\approx 11500 \mathrm{~K}$ is hampered by meridional flow. In stars hotter than this threshold, helium can settle and the superficial convection zone disappears, so that diffusion might occur in superficial regions of these stars. Recent evolutionary models of HB stars that include atomic diffusion calculated by Michaud et al. $(2008,2011)$ can reasonably well reproduce the abundance anomalies of several elements observed in blue HB stars. However, these models do not treat the atmospheric region in detail. Instead, they assume an outer superficial mixed zone of approximately $10^{-7} M_{\odot}$ below which separation occurs.

The detection of vertical stratification of some elements, especially iron, in the atmospheres of blue HB stars lends additional support to the scenario of atomic diffusion being active in these regions (Khalack et al. 2007, 2008, 2010), but is at variance with the mixed zone introduced by Michaud et al. (2008, 2011). Hui-Bon-Hoa et al. (2000) and LeBlanc et al. (2009) presented stellar atmosphere models of blue HB stars that include the effect of vertical abundance stratification on the atmospheric structure. These models estimate the vertical stratification of the elements caused by diffusion by assuming that an equilibrium solution (i.e. giving a nil diffusion-velocity) for each species is reached. Assuming a sudden onset of atomic diffusion near $11500 \mathrm{~K}$, these models predict photometric jumps and gaps (Grundahl et al. 1999; G1 in Ferraro et al. 1998) consistent with observations (see LeBlanc et al. 2010 for more details). The photometric changes, with respect to chemically homogeneous atmosphere models, are due to the modification of the atmospheric structure caused by the abundance stratification.

The $u$-jump described above can be clearly seen in the colour-magnitude diagram of NGC 288 shown in Fig. 1, where one also finds a group of stars with large (and unexplained) scatter in their $u$ magnitudes and $(u-y)$ colours around the jump region (triangles in Fig. 1). With maximum errors of 0.008 in $u$ and 0.003 in $y$ it is unlikely that their positions are caused by photometric errors. The evolutionary status of these stars is unclear. While their bright $u$ magnitudes are suggestive of radiative levitation, the effect would have to be extreme and some of them appear to lie on the cool side of the $u$-jump. Similar groups of unexpectedly bright stars can be found in other globular clusters (see M 2 and M92 in Grundahl et al. 1999), with NGC 288 presenting the most pronounced case. We discuss these stars in Sect. 8 in more detail.

A colour spread along the red giant branch in NGC 288 first reported by Yong et al. (2008) was identified as a split by Roh et al. (2011), which was confirmed by Carretta et al. (2011) and Monelli et al. (2013). In their excellent review on second and third parameters to explain the HB morphology, Gratton et al. (2010) estimated that a range in helium abundance of $\Delta Y=0.012$ would explain the temperature range of the $\mathrm{HB}$ in NGC 288. This is consistent with the helium range found more recently from the analysis of main-sequence photometry by Piotto et al. (2013). A variation in helium this small is unfortunately too small to be detected by our analysis.

\section{Observations}

The targets were selected from the Strömgren photometry of Grundahl et al. (1999). We selected 71 blue horizontal-branchstar candidates (see Fig. 1) and 17 red giants. Of the blue HB candidates three were found to be red HB stars and one had extremely noisy spectra. We here only discuss the observations of the 67 bona-fide hot HB stars (see Table 1 for their coordinates and photometric measurements).

The spectroscopic data were obtained between July 3 and 27, 2003 (date at the beginning of the night, see Table 2 for details) in Service Mode using the multi-object fibre spectrograph FLAMES+GIRAFFE (Pasquini et al. 2000), which is mounted at the UT2 Telescope of the VLT. The fibre systems MEDUSA1 and MEDUSA2 allow one to observe up to 132 objects simultaneously. We used the low spectroscopic resolution mode with the spectral ranges $3620-4081 \AA(L R 1, \lambda / \Delta \lambda=8000)$, 3964-4567 $(\mathrm{LR} 2, \lambda / \Delta \lambda=6400)$, and 4501-5078 $(\mathrm{LR} 3$, $\lambda / \Delta \lambda=7500)$. GIRAFFE had a $2 \mathrm{k} \times 4 \mathrm{k}$ EEV CCD chip ( $15 \mu \mathrm{m}$ pixel size), with a gain of $0.54 \mathrm{e}^{-} \mathrm{ADU}^{-1}$ and a read-outnoise of $3.2 \mathrm{e}^{-}$. Each night four screen flat-fields, five bias, and one ThAr wavelength calibration frame were observed. In addition to the daytime ThAr spectra that covered all fibres, we also observed simultaneous ThAr spectra during the science observations in five of the fibres. Dark exposures were not necessary, because the dark current of $2 \mathrm{e}^{-} \mathrm{h}^{-1}$ pixel $^{-1}$ is negligible.

\section{Data reduction}

The spectroscopic data were reduced using the girBLDRS software $^{1}$, version 1.10) and ESO MIDAS (see Drews 2005 for details). The two-dimensional bias and flat-field frames were averaged for each night. The averaged flat-fields were used to determine the positions and widths of the fibre spectra. Portions of 64-pixels of each fibre spectrum were averaged and fitted with a point spread function (PSF). A polynomial fit to the PSF parameters then provides the PSF for the whole frame, which is used for all extractions later on. One-dimensional flat-field spectra extracted using Horne's method (Horne 1986) are used to correct the extracted science spectra for spectrograph and detector signature. Because the flat-field spectra are not normalised, they still show the spectral signature of the flat-field lamp, which varies only slowly with wavelength, however.

The daytime Th-Ar wavelength calibration spectra were extracted for each fibre via a simple sum, the spectral lines were localised and fitted by an analytical model. The presence of simultaneous ThAr spectra in the science frames allowed us to

http://girbldrs. sourceforge.net/ 


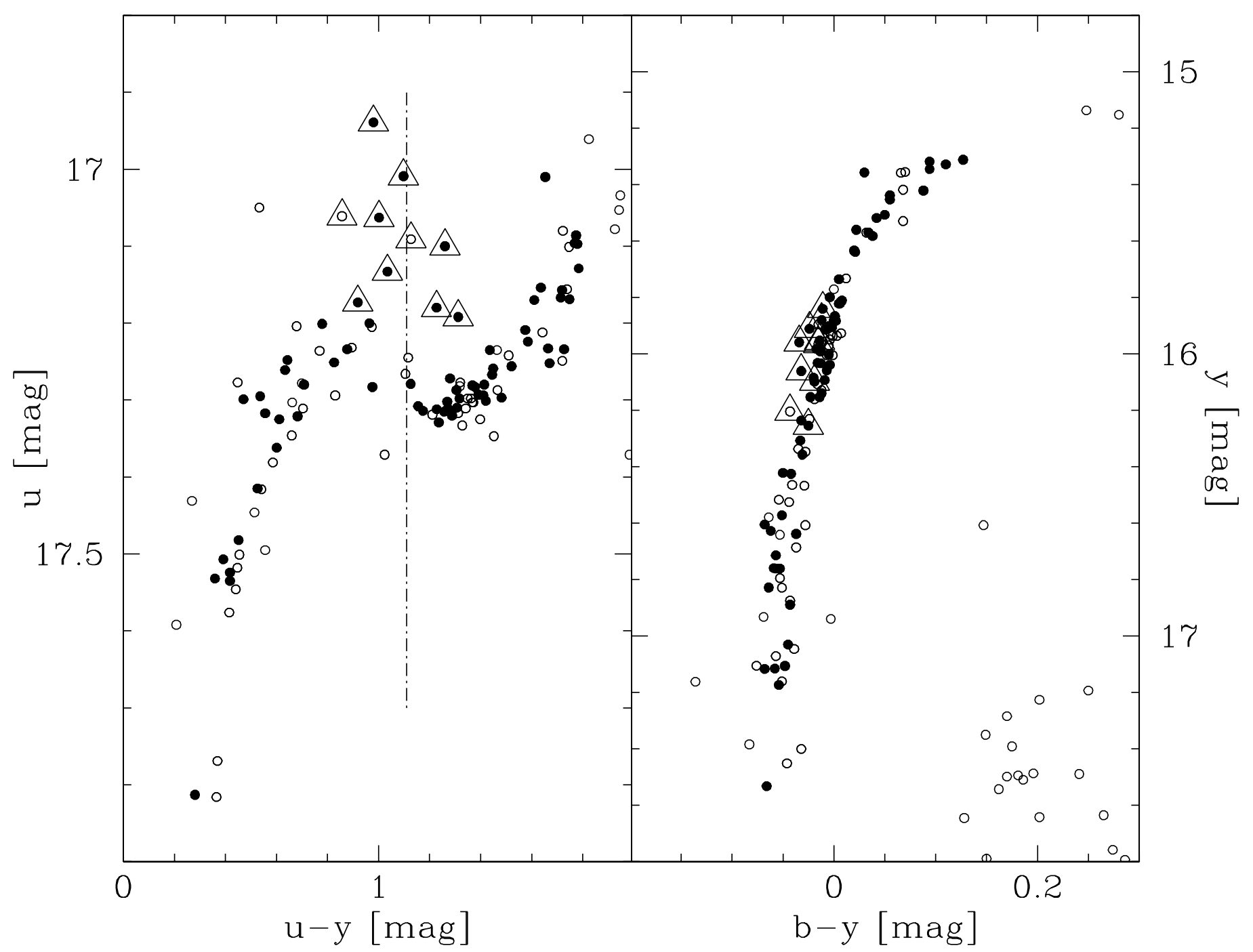

Fig. 1. Blue HB stars in NGC 288 as observed by Grundahl et al. (1999). The subsample, for which GIRAFFE spectra were observed, is marked by filled circles. The dot-dashed line in the left part marks the $u-y$ colour of the $u$-jump. Stars on the red side of the line do not show evidence for radiative levitation, while stars on the blue side do. The triangles mark overluminous stars around the $u$-jump (from red to blue: 122, 103, 127, $101,146,142,100,183)$.

refine the localisation and wavelength calibration of the science data by correcting for residual wavelength shifts between daytime and nighttime observations. The refinement was repeated until the difference between the observed ThAr positions and the laboratory ones was below $0.001 \mathrm{~km} \mathrm{~s}^{-1}$. The wavelength offsets obtained from the cross-correlation were then linearly interpolated across the CCD (spatial direction), and the interpolated offsets were applied to each fibre.

The raw science spectra were bias and flat-field corrected and finally rebinned to constant wavelength steps. The median of the sky signal obtained in 18 dedicated fibres was subtracted from the science data.

The spectra for a given star and setup were then normalised as described in Moehler et al. (2011), taking care to use only regions free from strong lines for the continuum definition. This normalisation allows us to exclude outlying pixels during the averaging of the spectra.

\section{Radial velocities}

After the barycentric correction, the observed spectra were first co-added (without further radial velocity correction) and fitted with stellar model spectra to obtain a first estimate of their effective temperatures, surface gravities, and helium abundances (see Sect. 5.2 for details). The individual spectra of each star were then cross-correlated (see Tonry \& Davis 1979 for more details) with the best-fitting synthetic spectrum derived this way. Only regions of hydrogen or helium lines were selected prior to the cross-correlation. The peak of the cross-correlation function was fitted with a Gaussian function to determine the radial velocity to sub-resolution accuracy. The velocity-corrected spectra were co-added and fitted with synthetic model atmospheres (see Sect. 5). In a second step, the best-fit synthetic spectra were then used to repeat the cross-correlation. The $1 \sigma$ errors in radial velocity for individual spectra range from $1.8 \mathrm{~km} \mathrm{~s}^{-1}$ to up to $10 \mathrm{~km} \mathrm{~s}^{-1}$ depending on the quality. The median error is $2.7 \mathrm{~km} \mathrm{~s}^{-1}$.

For each star, we calculated the standard deviation of the radial velocity measurements of the individual spectra. We then determined how many of the individual measurements deviate more than $1 \sigma$ from the mean. In none of the stars was this more than $30 \%$, which means that the scatter is consistent with a random distribution within the measurement error. We thus find no evidence for radial velocity variations that might indicate the presence of close binaries in our sample. We note that this 
absence of evidence should not be confused with an evidence of absence, that is, we cannot say anything about the frequency of binaries among our target stars, because neither our method nor our observations were tailored towards the detection of binaries. There is no evidence for field contamination in our sample either.

The radial velocities of the 67 stars range from $-48.6 \mathrm{~km} \mathrm{~s}^{-1}$ to $-36.9 \mathrm{~km} \mathrm{~s}^{-1}$ with a median value of $-42.6 \pm 2.7 \mathrm{~km} \mathrm{~s}^{-1}$, which is close to the average value of $-45.4 \mathrm{~km} \mathrm{~s}^{-1}$ given by Harris (1996) and to the recently published values of $-43.5 \mathrm{~km} \mathrm{~s}^{-1}$ (Székely et al. 2007, uncertainty $1-2 \mathrm{~km} \mathrm{~s}^{-1}$ ), $-46.15 \pm 2.55 \mathrm{~km} \mathrm{~s}^{-1}$ (Carretta et al. 2009b), and $-45.1 \pm 0.2 \mathrm{~km} \mathrm{~s}^{-1}$ (Lane et al. 2010).

Each spectrum was corrected to laboratory wavelength. As an additional safeguard against outliers, only a subset of pixel values around the median (11 out of 13 for LR1, 5 out of 7 for LR2, 3 out of 5 for LR3) was averaged. To allow a combination of the three wavelength ranges into one spectrum for fitting, the LR1 data were convolved with a Gaussian with a FWHM of $0.48 \AA$ so that we had the same resolution for all three regions.

\section{Atmospheric parameters}

\subsection{Determination from photometric data}

We used the Strömgren photometry of Grundahl et al. (1999) to derive a first estimate of the effective temperatures and surface gravities of stars redwards of the $u$-jump, similar to our work in Moehler et al. (2003). As reference we used theoretical colours from Kurucz (ATLAS9, 1993) for metallicities $[\mathrm{M} / \mathrm{H}]=-1.0$ and -1.5 . The metallicity $[\mathrm{Fe} / \mathrm{H}]$ of NGC 288 given by Carretta et al. (2009a) is -1.32 . Unfortunately, we have no $\mathrm{H} \beta$ photometry for the stars. Therefore, guided by the equations of Moon $\&$ Dworetsky (1985), we searched for a combination of colours that provides a rectangular grid in the $T_{\text {eff }}, \log g$ plane for the range $8000 \mathrm{~K} \leq T_{\text {eff }} \leq 12000 \mathrm{~K}$. We used the definition for $a_{0}$ from Moon \& Dworetsky, but in addition, we defined an index $s z$

$a_{0}=1.36 \cdot(b-y)+0.36 \cdot m_{1}+0.18 \cdot c_{1}-0.2448$
$s z=-0.07 \cdot(b-y)-m_{1}+0.1 \cdot c_{1}+0.1$.

As one can see from Fig. 2, $a_{0}$ correlates with $T_{\text {eff }}$ and $s z$ correlates with $\log g$ and $T_{\text {eff }}$. The photometric data were dereddened with $E_{B-V}=0.03$ (Carretta et al. 2000), using $E_{b-y}=0.75 \cdot E_{B-V}$.

To restrict the fitting range we used only $\log g$ between 3 and 4 and $T_{\text {eff }}$ between $8000 \mathrm{~K}$ and $12000 \mathrm{~K}$. We fitted a secondorder polynomial to the relation $T_{\text {eff }}\left(a_{0}\right)$, which yielded an rms deviation of $30 \mathrm{~K}$. For the surface gravities we fitted secondorder polynomials to the relation $\log g\left(a_{0}, s z\right)$, which yielded an rms deviation of 0.04 in $\log g$. Temperatures and surface gravities derived from these relations are listed in Table 3 and plotted in Fig. 3 for stars cooler than the $u$-jump.

The errors provided in Table 3 are a quadratic combination of errors from the photometric data, the uncertainty of the reddening in the $y$-band (estimated to be 0 . 02 ) and the error of the fit to the theoretical relations. To estimate the influence of metallicity we compared the results obtained for the two metallicities mentioned above. The differences in temperature vary almost linearly from $-0.1 \%$ at $8000 \mathrm{~K}$ to $+0.8 \%$ at $12000 \mathrm{~K}$, with the results from the more metal-poor models being hotter at the hot end. The differences are therefore well below the errors on the individual temperatures.

The differences in surface gravities for these two metallicities show a quadratic dependency on the effective temperature, varying from +0.02 at about $8600 \mathrm{~K}$ (with the surface gravities

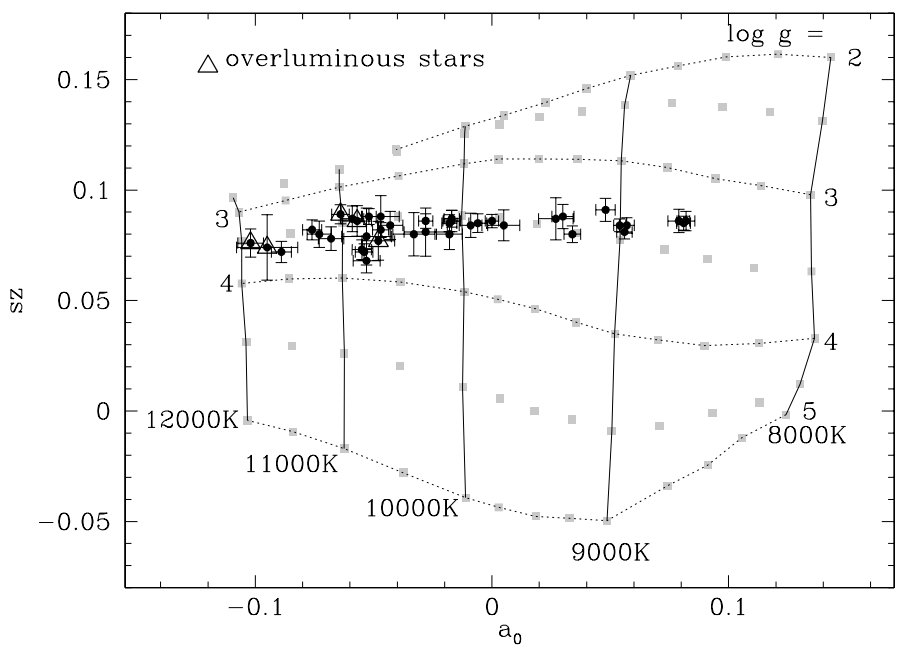

Fig. 2. Theoretical $a_{0}, s z$ grid as derived from Kurucz (1993) colours for $[\mathrm{Fe} / \mathrm{H}]=-1.5$. In addition, we show the positions of the cool blue HB stars (i.e. redward of the vertical line in Fig. 1) in this diagram as observed by Grundahl et al. (1999).

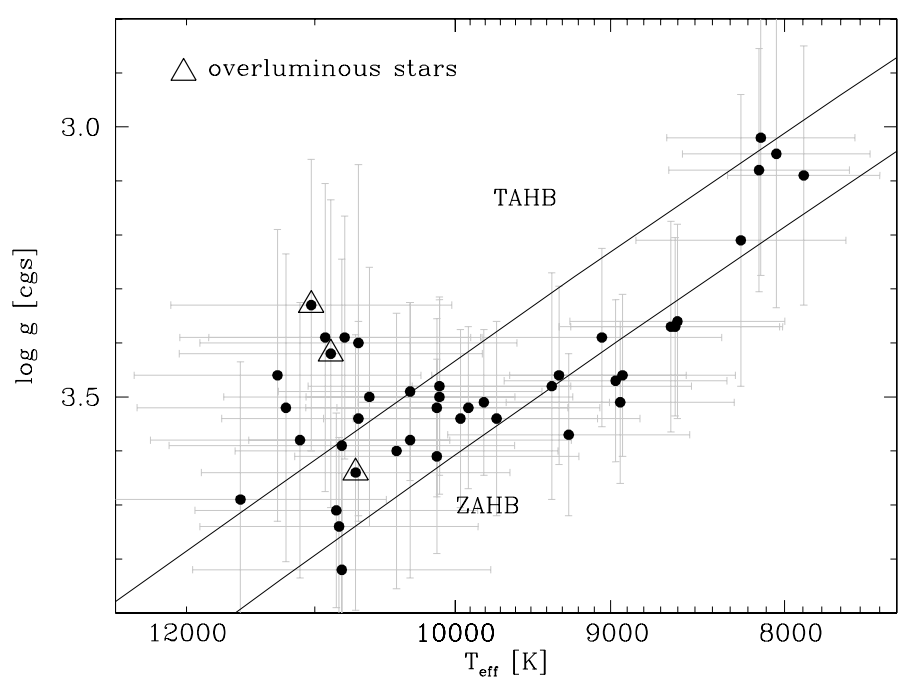

Fig. 3. Atmospheric parameters derived from the $a_{0}, s z$ indices for stars that do not show evidence for radiative levitation. For comparison we also show curves representing a canonical zero-age HB and terminalage HB from Moehler et al. (2003).

from the metal-poor models being higher at $8600 \mathrm{~K}$ than those from the more metal-rich models) to 0 at $9000 \mathrm{~K}$ and $11000 \mathrm{~K}$ via -0.02 at about $10000 \mathrm{~K}$. For temperatures below $8500 \mathrm{~K}$ the differences increase to -0.08 , but these stars are too cool to be analysed spectroscopically with our methods (see Sect. 5.2). Within the temperature range of interest for further analysis the differences are below the average errors of the surface gravities given in Table 3. For reasons of consistency between photometric and spectroscopic analysis we decided against averaging the photometric grids.

From the errors listed in Table 3 and the uncertainties due to the metallicity, we estimate typical errors in $T_{\text {eff }}$ and $\log g$ to be about $5 \%$ and 0.13 dex, respectively.

\subsection{Line profile fitting with homogeneous model spectra}

For stars redder than the $u$-jump, we computed model atmospheres with convection for $[\mathrm{M} / \mathrm{H}]=-1.5$ using ATLAS9 
Table 3. Temperatures and surface gravities for stars redder than the $u$-jump that do not show evidence of radiative levitation as derived from the $a_{0}$ and $s z$ parameters and from line profile fits for an assumed metallicity of $[\mathrm{M} / \mathrm{H}]=-1.5$.

\begin{tabular}{|c|c|c|c|c|c|c|c|}
\hline \multirow[t]{2}{*}{ ID } & \multicolumn{4}{|c|}{ Photometric parameters } & \multicolumn{3}{|c|}{ Spectroscopic parameters } \\
\hline & $\begin{array}{r}a_{0} \\
\text { [mmag] } \\
\end{array}$ & $\begin{array}{l}s z \\
{[\mathrm{mmag}]}\end{array}$ & $\begin{array}{r}T_{\text {eff }} \\
{[\mathrm{K}]} \\
\end{array}$ & $\log g$ & $\begin{array}{l}T_{\text {eff }} \\
{[\mathrm{K}]}\end{array}$ & $\log g$ & $\log \left(\frac{n_{\mathrm{He}}}{n_{\mathrm{H}}}\right)_{\text {LINFOR }}$ \\
\hline 52 & $+48 \pm 4$ & $91 \pm 5$ & $9100 \pm 380$ & $3.39 \pm 0.11$ & $8240 \pm 20$ & $2.87 \pm 0.02$ & \\
\hline 55 & $+124 \pm 4$ & $91 \pm 4$ & $8100 \pm 260$ & $3.08 \pm 0.15$ & & & \\
\hline 60 & $+134 \pm 6$ & $89 \pm 7$ & $8000 \pm 260$ & $3.05 \pm 0.19$ & & & \\
\hline 61 & $+151 \pm 3$ & $79 \pm 3$ & $7900 \pm 210$ & $3.09 \pm 0.16$ & & & \\
\hline 63 & $+125 \pm 5$ & $95 \pm 5$ & $8100 \pm 270$ & $3.02 \pm 0.17$ & & & \\
\hline 70 & $+82 \pm 4$ & $86 \pm 4$ & $8600 \pm 330$ & $3.36 \pm 0.12$ & $7990 \pm 20$ & $2.94 \pm 0.03$ & \\
\hline 72 & $+114 \pm 7$ & $86 \pm 8$ & $8200 \pm 300$ & $3.21 \pm 0.18$ & & & \\
\hline 74 & $+81 \pm 3$ & $85 \pm 3$ & $8600 \pm 320$ & $3.37 \pm 0.11$ & $7910 \pm 10$ & $2.94 \pm 0.04$ & \\
\hline 79 & $+79 \pm 5$ & $86 \pm 5$ & $8600 \pm 340$ & $3.37 \pm 0.13$ & $7990 \pm 10$ & $2.97 \pm 0.03$ & \\
\hline 81 & $+54 \pm 3$ & $84 \pm 3$ & $9000 \pm 350$ & $3.47 \pm 0.10$ & $8210 \pm 20$ & $2.98 \pm 0.02$ & \\
\hline 83 & $+34 \pm 4$ & $80 \pm 4$ & $9300 \pm 400$ & $3.57 \pm 0.10$ & $8360 \pm 30$ & $3.02 \pm 0.02$ & \\
\hline 86 & $+57 \pm 3$ & $84 \pm 4$ & $8900 \pm 360$ & $3.46 \pm 0.10$ & $8160 \pm 20$ & $3.00 \pm 0.02$ & \\
\hline 88 & $+30 \pm 5$ & $88 \pm 6$ & $9300 \pm 420$ & $3.46 \pm 0.11$ & $8390 \pm 30$ & $2.98 \pm 0.02$ & \\
\hline 89 & $+56 \pm 3$ & $81 \pm 3$ & $8900 \pm 360$ & $3.51 \pm 0.10$ & $8070 \pm 20$ & $2.93 \pm 0.03$ & \\
\hline 90 & $+27 \pm 7$ & $87 \pm 10$ & $9400 \pm 470$ & $3.48 \pm 0.14$ & $8450 \pm 30$ & $3.04 \pm 0.02$ & \\
\hline 96 & $+5 \pm 7$ & $84 \pm 7$ & $9700 \pm 500$ & $3.54 \pm 0.12$ & $8560 \pm 30$ & $3.07 \pm 0.02$ & \\
\hline 99 & $-17 \pm 6$ & $80 \pm 7$ & $10100 \pm 510$ & $3.61 \pm 0.12$ & $9130 \pm 260$ & $3.25 \pm 0.15$ & -0.6 \\
\hline 102 & $0 \pm 3$ & $86 \pm 3$ & $9800 \pm 440$ & $3.51 \pm 0.09$ & $9290 \pm 320$ & $3.38 \pm 0.18$ & -1.4 \\
\hline 103 & $-56 \pm 6$ & $86 \pm 7$ & $10900 \pm 590$ & $3.42 \pm 0.19$ & $9850 \pm 70$ & $3.34 \pm 0.04$ & -1.0 \\
\hline 106 & $-8 \pm 5$ & $84 \pm 6$ & $10000 \pm 490$ & $3.54 \pm 0.11$ & $9230 \pm 290$ & $3.33 \pm 0.17$ & -1.3 \\
\hline 107 & $-5 \pm 4$ & $85 \pm 4$ & $9900 \pm 460$ & $3.52 \pm 0.10$ & $9350 \pm 260$ & $3.39 \pm 0.15$ & -1.2 \\
\hline 111 & $-52 \pm 6$ & $68 \pm 6$ & $10800 \pm 570$ & $3.82 \pm 0.12$ & $9760 \pm 90$ & $3.44 \pm 0.05$ & -1.0 \\
\hline 113 & $-17 \pm 3$ & $85 \pm 4$ & $10100 \pm 470$ & $3.52 \pm 0.11$ & $9210 \pm 180$ & $3.31 \pm 0.11$ & -0.8 \\
\hline 114 & $-27 \pm 9$ & $81 \pm 11$ & $10300 \pm 600$ & $3.58 \pm 0.17$ & $9340 \pm 140$ & $3.33 \pm 0.08$ & -1.0 \\
\hline 115 & $-16 \pm 4$ & $86 \pm 5$ & $10100 \pm 480$ & $3.50 \pm 0.12$ & $9280 \pm 180$ & $3.34 \pm 0.10$ & -1.1 \\
\hline 118 & $-27 \pm 3$ & $86 \pm 3$ & $10300 \pm 480$ & $3.49 \pm 0.11$ & $9440 \pm 90$ & $3.38 \pm 0.05$ & -1.1 \\
\hline 119 & $-16 \pm 3$ & $87 \pm 4$ & $10100 \pm 470$ & $3.48 \pm 0.11$ & $9430 \pm 170$ & $3.39 \pm 0.09$ & -1.2 \\
\hline 120 & $-32 \pm 9$ & $80 \pm 10$ & $10400 \pm 600$ & $3.60 \pm 0.17$ & $9470 \pm 70$ & $3.39 \pm 0.04$ & -1.0 \\
\hline 122 & $-47 \pm 7$ & $77 \pm 9$ & $10700 \pm 590$ & $3.64 \pm 0.17$ & $9740 \pm 80$ & $3.40 \pm 0.04$ & -1.1 \\
\hline 127 & $-63 \pm 4$ & $89 \pm 4$ & $11000 \pm 550$ & $3.33 \pm 0.18$ & $9920 \pm 80$ & $3.37 \pm 0.04$ & -0.8 \\
\hline 143 & $-42 \pm 5$ & $84 \pm 6$ & $10600 \pm 550$ & $3.50 \pm 0.16$ & $9810 \pm 60$ & $3.45 \pm 0.03$ & -1.1 \\
\hline 145 & $-46 \pm 3$ & $82 \pm 3$ & $10700 \pm 520$ & $3.54 \pm 0.12$ & $9660 \pm 60$ & $3.42 \pm 0.03$ & -1.0 \\
\hline 147 & $-54 \pm 4$ & $73 \pm 5$ & $10800 \pm 550$ & $3.71 \pm 0.12$ & $9810 \pm 80$ & $3.43 \pm 0.04$ & -0.8 \\
\hline 149 & $-67 \pm 5$ & $78 \pm 5$ & $11100 \pm 590$ & $3.58 \pm 0.17$ & $9800 \pm 80$ & $3.41 \pm 0.04$ & -1.0 \\
\hline 151 & $-53 \pm 4$ & $72 \pm 4$ & $10800 \pm 540$ & $3.74 \pm 0.11$ & $9760 \pm 80$ & $3.43 \pm 0.04$ & -0.9 \\
\hline 154 & $-51 \pm 3$ & $88 \pm 4$ & $10800 \pm 520$ & $3.39 \pm 0.15$ & $10100 \pm 100$ & $3.51 \pm 0.04$ & -1.1 \\
\hline 156 & $-46 \pm 8$ & $88 \pm 10$ & $10700 \pm 610$ & $3.40 \pm 0.22$ & $9760 \pm 80$ & $3.37 \pm 0.04$ & -1.0 \\
\hline 157 & $-52 \pm 11$ & $79 \pm 13$ & $10800 \pm 670$ & $3.59 \pm 0.23$ & $9580 \pm 90$ & $3.34 \pm 0.05$ & -1.1 \\
\hline 169 & $-88 \pm 4$ & $72 \pm 5$ & $11600 \pm 600$ & $3.69 \pm 0.17$ & $10400 \pm 100$ & $3.53 \pm 0.05$ & -1.0 \\
\hline 176 & $-72 \pm 5$ & $80 \pm 6$ & $11200 \pm 600$ & $3.52 \pm 0.19$ & $10300 \pm 100$ & $3.52 \pm 0.05$ & -1.0 \\
\hline 180 & $-75 \pm 4$ & $82 \pm 5$ & $11300 \pm 580$ & $3.46 \pm 0.18$ & $10600 \pm 80$ & $3.60 \pm 0.04$ & -1.1 \\
\hline
\end{tabular}

Notes. The errors for the photometric results are derived from the errors of $a_{0}$ and $s z$ in combination with the errors of the fits and an uncertainty of 0.02 for the reddening (see Sect. 5.1 for details). The errors for the spectroscopic results are derived from the $\chi^{2}$ fit (see Sect. 5.2 for details) and are only statistical errors. In addition, we list the helium abundance as determined with LINFOR (see Sect. 6 for details).

(Kurucz 1993) and used Lemke's version of the LINFOR program (developed originally by Holweger, Steffen, and Steenbock at Kiel University) to compute a grid of theoretical spectra that include the Balmer lines $\mathrm{H} \alpha$ to $\mathrm{H}_{22}$ and the $\mathrm{He}$ I and $\mathrm{He}$ II lines. The grid covers the range $7500 \mathrm{~K} \leq T_{\text {eff }} \leq 12000 \mathrm{~K}$, $2.5 \leq \log g \leq 5.0$, and $-3.0 \leq \log \frac{n_{\mathrm{He}}}{n_{\mathrm{H}}} \leq-1.0$. For stars bluer than the $u$-jump we used model atmospheres computed for $[\mathrm{M} / \mathrm{H}]=+0.5$ (see Moehler et al. 2000 for details). From an extrapolation of the LTE/NLTE threshold for subdwarf B stars (Napiwotzki 1997) we assumed that LTE is a valid approximation here as well. To establish the best fit to the observed spectra, we used the fitsb2 routines developed by Napiwotzki et al. (2004), which employ a $\chi^{2}$ test. The $\sigma$ necessary to calculate $\chi^{2}$ was estimated from the noise in the continuum regions of the spectra. The fit program normalises synthetic model spectra and observed spectra using the same points for the continuum definition.

While the errors were obtained via boot strapping and should therefore be rather realistic, they do not include possible systematic errors due to flat-field inaccuracies or imperfect sky subtraction, for instance. The true errors in $T_{\text {eff }}$ are probably close to those from photometry, that is, $5 \%$, and the true errors in $\log g$ are probably about 0.1 .

Because the $\chi^{2}$ fit of the line profile can lead to ambiguous results for effective temperatures close to the Balmer maximum, the results from Sect. 5.1 were taken as initial parameters for the spectral line profile fitting procedure.

In Table 3 we list the results obtained from fitting the Balmer lines $\mathrm{H} \beta$ to $\mathrm{H}_{10}$ (excluding $\mathrm{H} \epsilon$ to avoid the $\mathrm{Ca}$ II $\mathrm{H}$ line) in the cool stars. For the cool stars we did not fit the helium lines 
Table 4. Temperatures, surface gravities, and helium abundances from line profile fits for stars bluer than the $u$-jump that show evidence of radiative levitation.

\begin{tabular}{rrrrr}
\hline \hline ID & $T_{\text {eff }}$ & $\log g$ & \multicolumn{2}{c}{$\log \left(\frac{n_{\text {He }}}{n_{\mathrm{H}}}\right)$} \\
& {$[\mathrm{K}]$} & & fitsb2 & LINFOR \\
\hline 100 & $11400 \pm 100$ & $3.65 \pm 0.03$ & $-2.23 \pm 0.11$ & -2.14 \\
101 & $11400 \pm 100$ & $3.78 \pm 0.03$ & $-1.95 \pm 0.11$ & -1.93 \\
142 & $11400 \pm 100$ & $3.72 \pm 0.03$ & $-2.98 \pm 0.12$ & -2.76 \\
146 & $11600 \pm 100$ & $3.81 \pm 0.03$ & $-2.06 \pm 0.10$ & -2.02 \\
179 & $11600 \pm 100$ & $3.77 \pm 0.03$ & $-2.37 \pm 0.10$ & -2.14 \\
183 & $12300 \pm 100$ & $3.93 \pm 0.03$ & $-2.27 \pm 0.12$ & -2.36 \\
187 & $11700 \pm 100$ & $3.84 \pm 0.03$ & $-2.74 \pm 0.13$ & -2.87 \\
195 & $12400 \pm 100$ & $3.86 \pm 0.03$ & $-2.55 \pm 0.15$ & -2.51 \\
196 & $12300 \pm 100$ & $3.91 \pm 0.03$ & $-2.67 \pm 0.18$ & -2.49 \\
199 & $12400 \pm 100$ & $3.94 \pm 0.03$ & $-2.79 \pm 0.18$ & -2.84 \\
212 & $12900 \pm 100$ & $3.93 \pm 0.02$ & $-2.66 \pm 0.12$ & -2.57 \\
213 & $13100 \pm 100$ & $3.97 \pm 0.03$ & $-2.61 \pm 0.15$ & -2.68 \\
216 & $12900 \pm 100$ & $3.94 \pm 0.02$ & $-2.66 \pm 0.14$ & -2.71 \\
221 & $13200 \pm 100$ & $3.97 \pm 0.03$ & $-2.67 \pm 0.13$ & -2.60 \\
228 & $13800 \pm 100$ & $4.00 \pm 0.03$ & $-2.43 \pm 0.17$ & -2.43 \\
230 & $13800 \pm 100$ & $4.06 \pm 0.03$ & $-2.28 \pm 0.10$ & -2.34 \\
231 & $13300 \pm 100$ & $4.00 \pm 0.02$ & $-2.72 \pm 0.09$ & -2.55 \\
240 & $13300 \pm 100$ & $3.99 \pm 0.03$ & $-2.71 \pm 0.11$ & -2.61 \\
242 & $14300 \pm 100$ & $4.03 \pm 0.03$ & $-2.37 \pm 0.13$ & -2.28 \\
243 & $14400 \pm 100$ & $4.09 \pm 0.04$ & $-2.52 \pm 0.15$ & -2.43 \\
275 & $15400 \pm 200$ & $4.16 \pm 0.03$ & $-2.44 \pm 0.15$ & -2.27 \\
288 & $15200 \pm 200$ & $4.14 \pm 0.04$ & $-2.19 \pm 0.11$ & -2.16 \\
292 & $15100 \pm 100$ & $4.23 \pm 0.03$ & $-2.92 \pm 0.07$ & -2.80 \\
300 & $15400 \pm 200$ & $4.14 \pm 0.04$ & $-2.21 \pm 0.10$ & -2.19 \\
304 & $16900 \pm 200$ & $4.29 \pm 0.04$ & $-2.57 \pm 0.12$ & -2.46 \\
347 & $16400 \pm 200$ & $4.38 \pm 0.04$ & $-2.11 \pm 0.09$ & -2.06 \\
\hline & & & & \\
\hline & & & & \\
& & & & \\
& & &
\end{tabular}

Notes. The errors are derived from the $\chi^{2}$ fit (see Sect. 5.2 for details) and are only statistical errors. In addition, we list the helium abundance as determined with LINFOR (see Sect. 6 for details).

because they are very weak and tend to produce spurious results. We verified, however, that the helium lines predicted by the model spectra with solar helium abundance did reproduce the observations. The reddest stars that showed many strong metal lines $(55,60,61,63,72)$ were omitted from the line profile fits because our model spectra contain only $\mathrm{H}$ and $\mathrm{He}$ lines. In addition, we fitted the He I lines at $\lambda \lambda 4026 \AA$, $4388 \AA, 4472 \AA$, and $4922 \AA$ for stars hotter than $11500 \mathrm{~K}$.

Figure 4 shows a gap at $8500 \mathrm{~K} \leq T_{\text {eff }} \leq 9000 \mathrm{~K}$ in the atmospheric parameters derived from line profile fitting, which was first noted and discussed by Moehler et al. (2003) for observations of $\mathrm{HB}$ stars in M 13. Stars populating this region in Fig. 3 close to the zero-age HB (ZAHB) are located at about $8000 \mathrm{~K}$ in Fig. 4, above the terminal-age HB (TAHB). Prompted by the referee, we treated a homogeneous model spectrum calculated for $T_{\text {eff }}=8800 \mathrm{~K}, \log g=3.35$ and $[\mathrm{M} / \mathrm{H}]=-1.5$ as described in Sect. 6.3 to simulate an observed spectrum. Varying the starting values of the fit from $8000 \mathrm{~K}$ to $9500 \mathrm{~K}$ for $T_{\text {eff }}$ and 3.2 to 3.6 for $\log g$ did not affect the fit results, which remained at $T_{\text {eff }}=8841 \mathrm{~K}$ and $\log g=3.35$. Varying the FWHM of the Gaussian with which the model spectra are convolved before fitting from $0.6 \AA$ to $0.75 \AA$ resulted in $T_{\text {eff }}$ between $8818 \mathrm{~K}$ and $8862 \mathrm{~K}$ and $\log g$ between 3.34 and 3.36. We noted, however, that the resolution of the observed data seems to vary slightly with wavelength - at the blue end the predicted line profiles are very slightly wider and deeper than the observed ones, while for $\mathrm{H} \beta$ the observed line core is slightly more narrow and deep. To rule out possible small variations in resolution across the wavelength

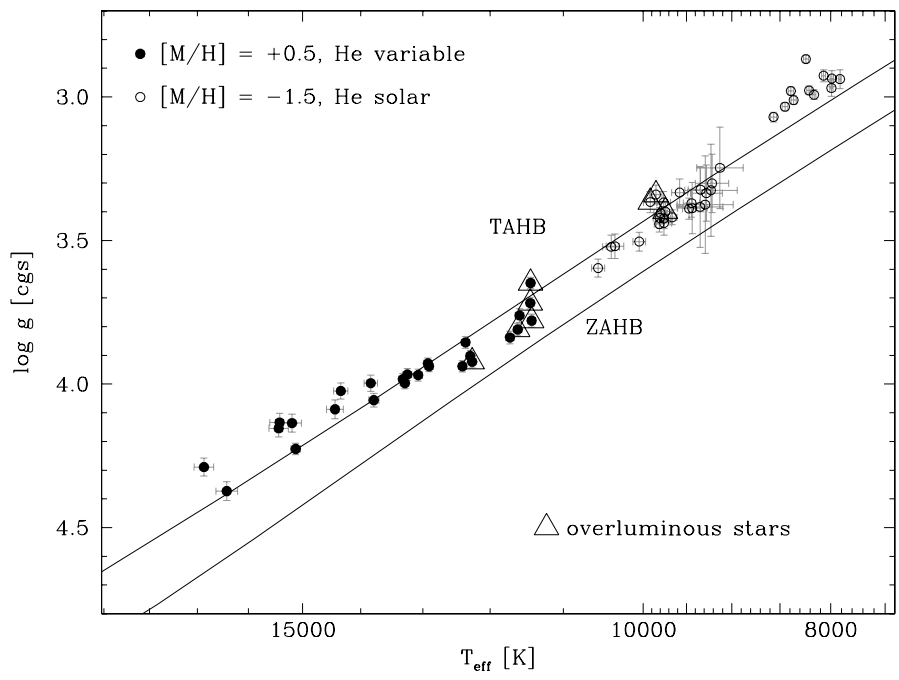

Fig. 4. Atmospheric parameters derived from the line profile fitting for stars hotter (full symbols) and cooler (open symbols) than the $u$-jump. For comparison we also show a canonical zero-age HB and terminalage HB from Moehler et al. (2003).

range as the cause for the gap, we convolved the observed spectra to a resolution of $2.5 \AA$, which is close to the resolution of the data of Moni Bidin et al. $(2007,2009,2011)$ and Salgado et al. (2013), which do not show such a gap in their results, although they used the same analysis methods as we did. Unfortunately, the gap remains in the results of the line profile fitting. Using model atmospheres with or without convection did not affect the gap either.

The stars in Fig. 4 can be split into two groups with respect to their position relative to the evolutionary sequences: stars with effective temperatures between $9000 \mathrm{~K}$ and roughly $14000 \mathrm{~K}$ lie between the ZAHB and the TAHB, while stars hotter or cooler than this temperature range lie above the TAHB.

To determine how the parameters derived for the hot HB stars in NGC 288 differ from those obtained for other clusters and from the theoretical predictions we calculated the difference between the derived surface gravity and that on the ZAHB for the given temperature. Figure 5 compares our results with those obtained for M 80, NGC 5986 (Moni Bidin et al. 2009), NGC 6752 (Moni Bidin et al. 2007), M 22 (Salgado et al. 2013), and $\omega$ Cen (Moehler et al. 2011; Moni Bidin et al. 2011). Here the NGC 288 results resemble those from $\omega$ Cen for temperatures lower than $9000 \mathrm{~K}$ or higher than about $14000 \mathrm{~K}$, while they coincide with the other clusters for temperatures between $9000 \mathrm{~K}$ and $14000 \mathrm{~K}$.

Using the spectroscopically determined atmospheric parameters, $y$-magnitudes, and the distance modulus of the globular cluster $\left((m-M)_{\mathrm{V}}=14 \mathrm{~m} .95\right.$, Carretta et al. 2000), we derived the masses shown in Fig. 6 using the equation

$$
\frac{M}{M_{\odot}}=\frac{3.6 \times 10^{-7}}{\pi \cdot g_{\odot} \cdot R_{\odot}[\mathrm{pc}]} \times 10^{-0.4 \cdot\left(y-(m-M)_{V}-y_{\mathrm{th}}\right)}
$$

where $y_{\text {th }}$ is the theoretical $y$ magnitude at the stellar surface from Kurucz (1993) and $R_{\odot}$ is the solar radius in parsec. Obviously, the masses are systematically too low, except for stars just hotter than the $u$-jump. Compared with results from other clusters, the masses of the stars in NGC 288 show a similar behaviour as the surface gravities with respect to HB stars in other globular clusters. 
S. Moehler et al.: Hot horizontal-branch stars in NGC 288 - diffusion and stratification effects

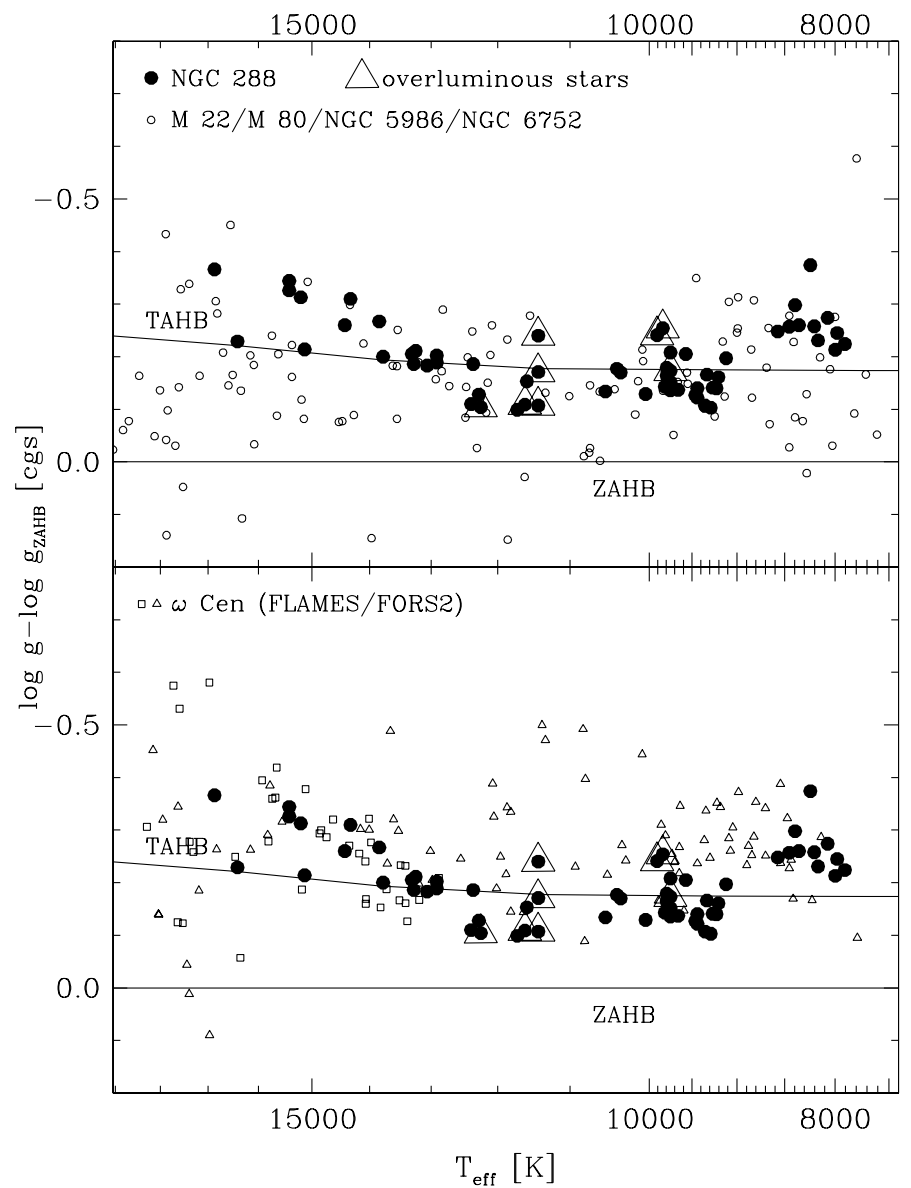

Fig. 5. Surface gravity relative to the zero-age HB at the given effective temperature. For comparison we also show a canonical zero-age and terminal-age HB from Moehler et al. (2003). In the upper plot we also show results from FORS2 observations of hot HB stars in M 80, NGC 5986 (Moni Bidin et al. 2009), NGC 6752 (Moni Bidin et al. 2007), and M 22 (Salgado et al. 2013). In the lower plot we also provide the results obtained from FLAMES and FORS2 observations of hot HB stars in $\omega$ Cen (Moehler et al. 2011, small squares; Moni Bidin et al. 2011, small triangles).

\section{Abundances}

We used the parameters derived in Sect.5.2 for the following abundance analysis.

\subsection{Helium}

Helium abundances were already derived during the determination of effective temperatures and surface gravities. In addition, we determined them (together with abundances for other elements) via spectrum synthesis using the abundance-fitting routine of LINFOR (see Sect.6.2 for details). The resulting abundances are listed in Tables 3 and 4. For the cool stars the abundances should be treated with caution because the helium lines are rather weak. However, the average fitted helium abundance $\log \frac{n_{\mathrm{He}}}{n_{\mathrm{H}}}$ of -1.03 agrees well with our assumption of solar helium abundance for cool stars. For hot stars the helium abundances derived with LINFOR are in general slightly higher than those derived with fitsb2, but in $80 \%$ of the cases the difference is smaller than the error provided by fitsb2. On average, the LINFOR abundances are higher by $0.06 \mathrm{dex}$, that is, much smaller than the error of the fitsb2 results.

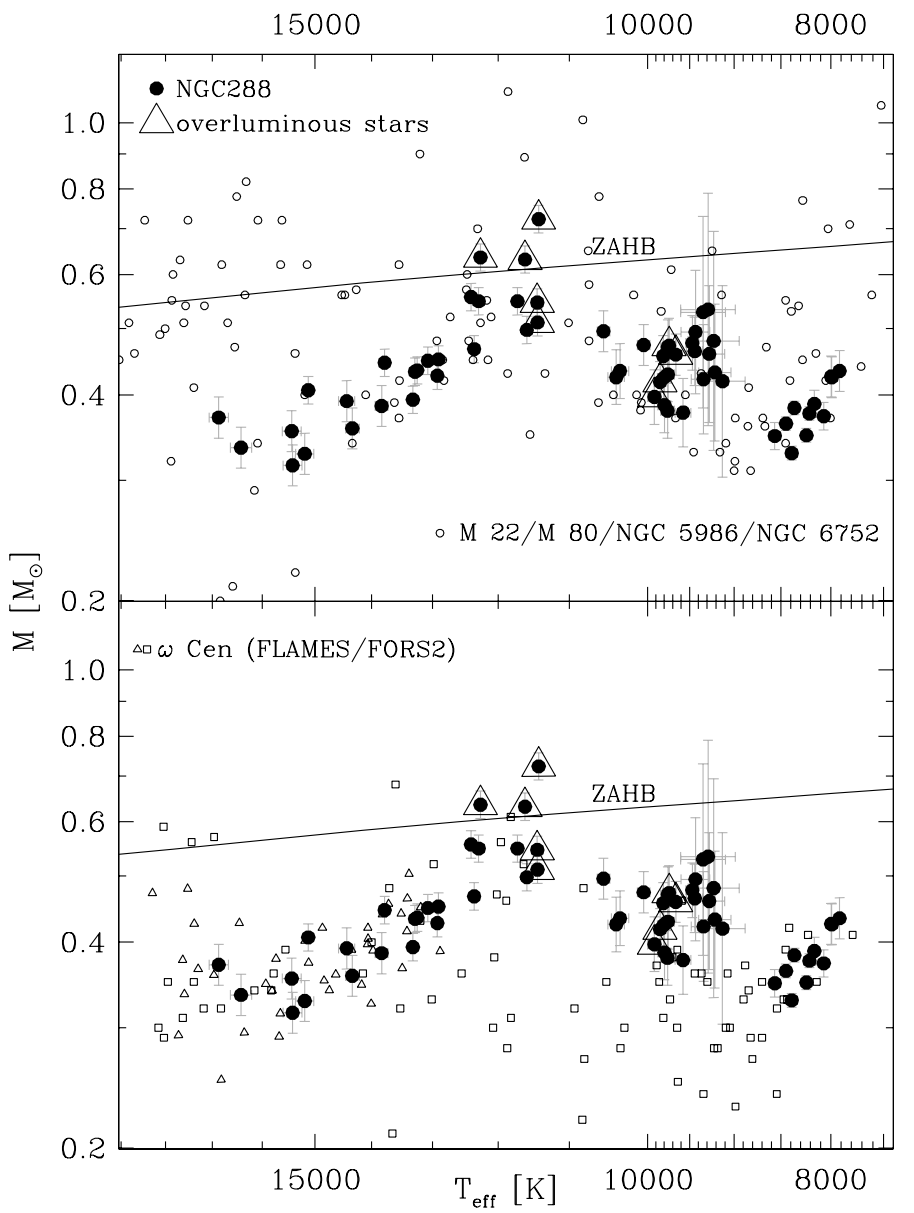

Fig. 6. Masses from line profile fits. For comparison we also show a canonical zero-age HB from Moehler et al. (2003). In the upper plot we also show results from FORS2 observations of hot HB stars in M 80, NGC 5986 (Moni Bidin et al. 2009), NGC 6752 (Moni Bidin et al. 2007), and M 22 (Salgado et al. 2013). In the lower plot we also provide the results obtained from FLAMES and FORS2 observations of hot HB stars in $\omega$ Cen (Moehler et al. 2011, small squares; Moni Bidin et al. 2011, small triangles).

Because helium abundances have been derived for HB stars in many globular clusters with similar methods and model spectra as we used here, we compared our results with published data in Fig. 7. The helium abundances of Moni Bidin et al. (2007, 2009, 2012) and Moehler et al. (2000, 2003) were derived from low-resolution data with a resolution of 2.6-3.4 $\AA$. The abundances of Behr (2003) were derived from data with a resolution of $0.1 \AA$, while the data used here have a resolution of about $0.7 \AA$. Between $11000 \mathrm{~K}$ and $13500 \mathrm{~K}$ the abundances show a clear correlation with the resolution of the data from which they were derived - the abundances decrease with increasing resolving power. This behaviour had been noticed already by Moni Bidin et al. (2012). This may be caused by the fact that at these relatively cool temperatures the helium lines are rather weak, which together with the helium deficiency makes them hard to fit. For higher temperatures the abundances from the different sources overlap, with the exception of the hottest star of Behr (2003). Due to the small number of results from highresolution spectroscopy it is not clear whether the resolution effect persists to higher temperatures. For a comparison with predictions from diffusion theory see Sect. 6.4. 


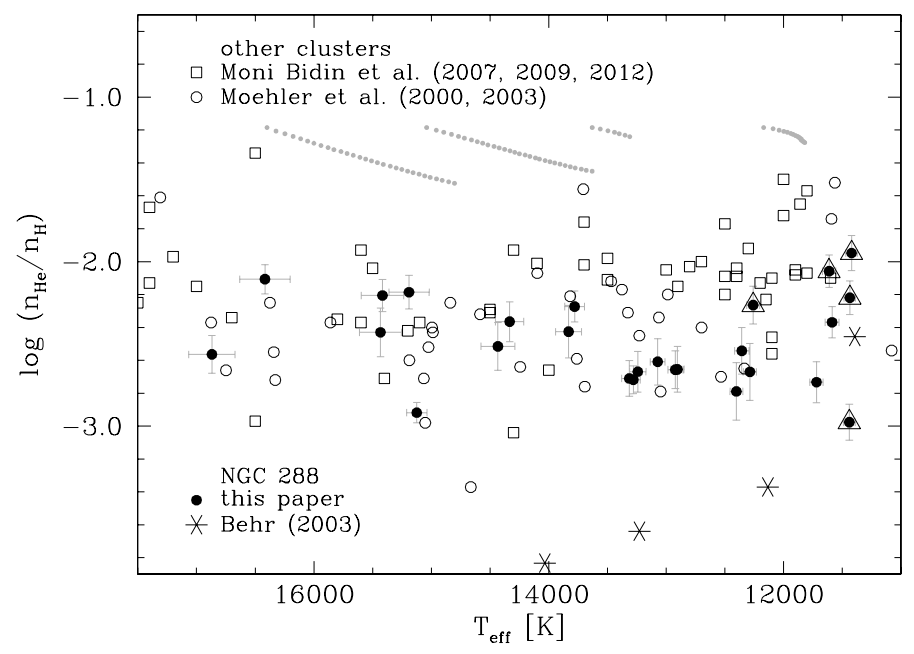

Fig. 7. Helium abundances for hot HB stars in M80, NGC 5986, NGC 6752, and $\omega$ Cen (Moni Bidin et al.), M3, M 13, and NGC 6752 (Moehler et al.) and NGC 288. The grey dots mark the abundances predicted for various HB ages from diffusion theory (with an ad hoc surface-mixing zone, Michaud et al. 2011, see Sect. 6.4 for details). The tracks have dots every $3 \mathrm{Myr}$.

\subsection{Heavy elements}

Although the spectra have only medium resolution, we estimated abundances via spectrum synthesis using the abundance-fitting routine of LINFOR to verify whether our assumptions about the overall increase in heavy elements were roughly correct. We used the line lists from Kurucz (1993) and simultaneously determined abundances for He I, Mg II, Si II, P II, Ti II, Mn II, Fe II, and Ni II. When the fitted abundances did not change anymore from one iteration to the next, we visually verified that the spectrum had indeed been well reproduced. The results are shown in Fig. 8. The LINFOR abundance-fitting routine only provides an overall error, but the scatter of abundances below $11000 \mathrm{~K}$ gives an idea of the minimum uncertainties, because we do not expect the abundances shown in Fig. 8 to vary in these cool stars. For stars hotter than the $u$-jump additional uncertainties arise because we used homogeneous model atmospheres to analyse stars affected by diffusion.

The results for P II, Ti II, Mn II, Fe II, and Ni II approximately agree with our assumption of $[\mathrm{M} / \mathrm{H}]=+0.5$ for stars hotter than the $u$-jump, while $\mathrm{Mg}$ II and Si II, on the other hand, are rather lower than our assumed abundances. However, since the atmospheric structure depends more on iron than on the light elements, we took these results to indicate that our assumptions were reasonable. For the cool stars, $\mathrm{Mg}$ II and Ti II seem to show an unexpected gradient with temperature, but it is unclear whether this is significant.

Our results show some differences compared with those obtained for NGC 288 by Behr (2003). Behr (2003) found no clear dependence of the $\mathrm{Mg}$ abundance relative to $T_{\mathrm{eff}}$ for the three clusters studied with a sufficient number of stars with $T_{\text {eff }}$ above the $u$-jump, while a trend appears to be present in our results for NGC 288. For Si and Mn Behr found significantly lower abundances than we do, which might be an effect of instrumental resolution (see Sect. 6.1). The observed abundances shown in Fig. 8 are provided at the CDS.

\subsection{Abundances predicted by stratified model spectra}

To compare the results from our observed spectra with the predictions from stratification theory we also determined

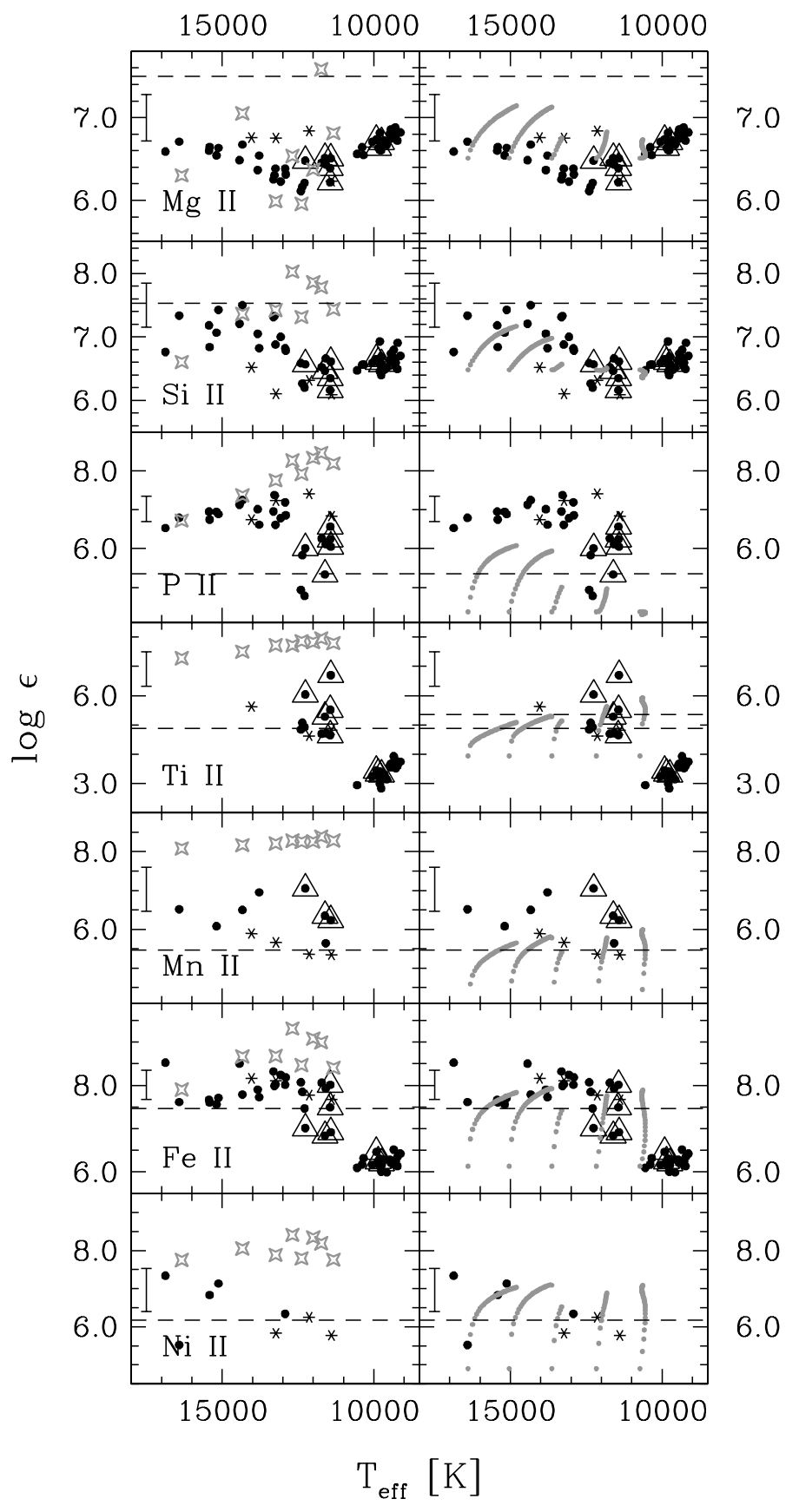

Fig. 8. Abundances derived via spectrum synthesis for all stars hotter than $9000 \mathrm{~K}$. The triangles mark the same stars as in Fig. 1. The asterisks mark the results from Behr (2003). The bars at $17500 \mathrm{~K}$ mark the average error bars of Behr (2003) for NGC 288. Our errors are probably not smaller. The dashed lines mark the solar abundances. The fourpointed stars mark the abundances derived for stratified model spectra (left column, see Sect. 6.3 for details), which indicate the equilibrium abundances achievable by diffusion. The grey dots (right column) mark the abundances predicted for various $\mathrm{HB}$ ages from diffusion theory (with an ad hoc surface-mixing zone, Michaud et al. 2011, see Sect. 6.4 for details). The tracks have dots every $1 \mathrm{Myr}$.

abundances from model spectra of LeBlanc et al. (2009), which include abundance stratification due to diffusion. These models self-consistently calculate the structure of the atmosphere with the vertical abundance stratification. The abundances of the individual elements are calculated in each layer of the atmosphere while assuming equilibrium (nil diffusion-velocity). This 
Table 5. Temperatures and surface gravities obtained from fitting stratified model spectra with homogeneous model spectra for $[\mathrm{M} / \mathrm{H}]=+1$ (see Sect. 6.3 for details).

\begin{tabular}{ccr|rr}
\hline \hline & Model & \multicolumn{2}{|c}{ Fit } \\
& $T_{\text {eff }} / \mathrm{K}$ & $\log g$ & $T_{\text {eff }} / \mathrm{K}$ & $\log g$ \\
\hline $\mathrm{A}$ & 12000 & 3.5 & $11500 \pm 100$ & $3.07 \pm 0.05$ \\
$\mathrm{~B}$ & 12000 & 4.0 & $11700 \pm 100$ & $3.60 \pm 0.06$ \\
$\mathrm{C}$ & 12000 & 4.5 & $11300 \pm 100$ & $3.94 \pm 0.06$ \\
$\mathrm{D}$ & 13000 & 3.5 & $12200 \pm 100$ & $3.08 \pm 0.05$ \\
$\mathrm{E}$ & 13000 & 4.0 & $12000 \pm 100$ & $3.48 \pm 0.05$ \\
$\mathrm{~F}$ & 13000 & 4.5 & $12400 \pm 100$ & $4.00 \pm 0.05$ \\
$\mathrm{G}$ & 14000 & 4.0 & $12700 \pm 100$ & $3.47 \pm 0.04$ \\
$\mathrm{H}$ & 14000 & 4.5 & $13200 \pm 100$ & $4.01 \pm 0.04$ \\
$\mathrm{I}$ & 16000 & 4.5 & $14300 \pm 100$ & $3.98 \pm 0.04$ \\
$\mathrm{~J}$ & 18000 & 5.0 & $16300 \pm 100$ & $4.52 \pm 0.03$ \\
\hline
\end{tabular}

Notes. The errors are derived from the $\chi^{2}$ fit (see Sect. 5.2 for details) and are only statistical errors.

leads to vertical abundance stratification and modifies the atmospheric structure. First we compared the observed metal lines with those predicted by stratification models and found that the observed lines were much weaker than predicted by these models (see Fig. 9).

As discussed in LeBlanc et al. (2009), the abundances of some elements ( $\mathrm{Fe}$, for example) predicted by the models can be overestimated because in a real star the diffusion process is a time-dependent phenomenon. Even though the radiative forces on a given element can theoretically support a very large overabundance, this situation may be hampered by various factors. The equilibrium solution used in these models may therefore not be reached. For instance, in a real star, such a large quantity of atoms might not be able to surface due to the diffusion that takes place below the stellar atmosphere. In addition, the abundances of other elements (such as helium) that have relatively weak radiative accelerations can be underestimated by these models.

Next we convolved the stratified model spectra to the same resolution as our observed data and multiplied them with a spectrum of average 1 and rms of 0.0125 , resulting in a signal-tonoise ratio of 80 . Then we determined their effective temperatures and surface gravities in the same way as described in Sect. 5.2, except that we used a model grid with $[\mathrm{M} / \mathrm{H}]=+1$, since the lines in the stratified model spectra were much stronger than in the observed spectra (see Fig. 9). The resulting parameters are given in Table 5 and clearly show that the analysis of stratified model spectra with homogeneous model spectra yields lower temperatures and surface gravities, with the difference in temperature increasing with increasing temperature.

Using the parameters in the right column of Table 5, we then determined abundances for the model spectra with fitted log $g$ above 3.45 as expected for stars on or near the ZAHB at the fitted temperatures, that is, all models except $\mathrm{A}$ and $\mathrm{D}$. We alternated between simultaneously fitting the ions Mg II, Si II, P II, S II, A II, Ti II, V II, Cr II, and simultaneously fitting the ions Mn II, Fe I, Fe II, Co II, Ni II, Sr II, and Zr II, until the fit did not improve any more. Then we again visually checked that most of the spectrum had been reproduced. Typically, we found that some lines were not fit well, but that was to be expected since the abundance distribution in the model atmospheres creating these spectra is far from homogeneous, which results in a very different atmospheric structure. The results of these fits are marked by four-pointed stars in the left column of Fig. 8. As already suggested by the comparison shown in Fig. 9, the abundances derived from the

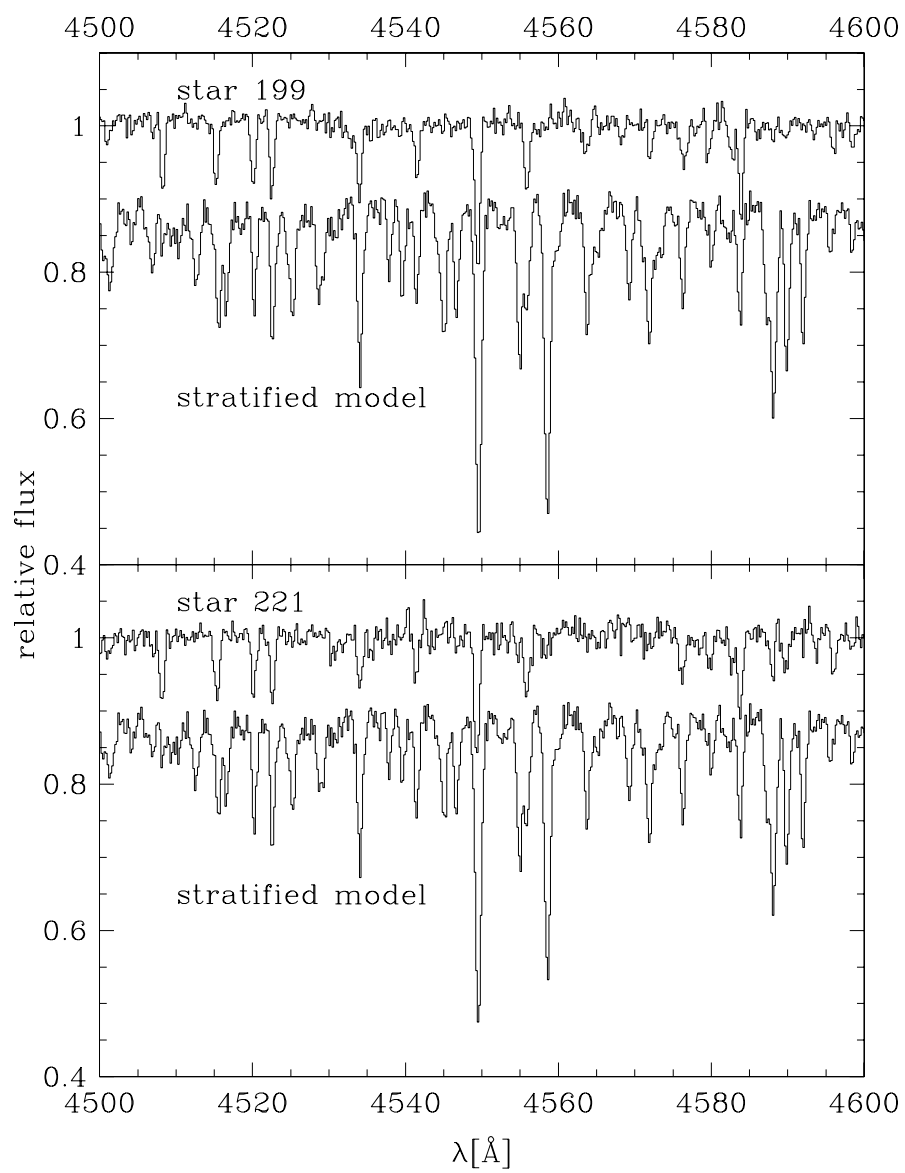

Fig. 9. Normalised spectra for stars 199 (upper panel, $12400 \mathrm{~K}$ ) and 221 (lower panel, $13400 \mathrm{~K}$ ) compared with stratified model spectra $\mathrm{F}$ (upper panel) and $\mathrm{H}$ (lower panel), which have fitted parameters close to those of the observed spectra. For clarity, the stratified model spectra have been offset by 0.1 from the observed spectra (see Tables 4 and 5).

stratified spectra are generally higher than those derived from the observed spectra, with He I (virtually absent from stratified model atmospheres) and Mg II being the exceptions.

\subsection{Abundances predicted by diffusion models}

It is also possible to compare our abundance results with stellar evolution models computed including the effects of atomic diffusion (Michaud et al. 2008, 2011). These models followed the evolution from the zero-age main sequence, treating in detail atomic diffusion inside the star. On the HB, they predicted large overabundances of metals, often larger than observed above $11000 \mathrm{~K}$ (similar to the problems found above for the stratified model atmospheres). A surface mixed mass of around $10^{-7} M_{\odot}$ was needed to reduce the expected anomalies to values observed in HB stars of a number of clusters as well as in sdB stars. However, the introduction of a surface mixing layer leads to a vertically homogeneous atmosphere. This contradicts the observed vertical stratification of certain elements, including iron, in some blue HB stars (Khalack et al. 2007, 2008, 2010). The tracks shown in Fig. 8 are taken from Michaud et al. (2011). These tracks start with the original cluster abundances at the 
ZAHB and cover the first third of horizontal branch evolution, which lasts some $100 \mathrm{Myr}^{2}$.

Figure 8 shows that the abundances change more rapidly during the first $10 \mathrm{Myr}$ than during the following $20 \mathrm{Myr}$ for all species that become overabundant. This is largely caused by a reduction of the radiative accelerations as the concentrations increase. The abundance increase is also expected to be slow during the following $70 \mathrm{Myr}$ for these species. The $\mathrm{Si}, \mathrm{Ti}, \mathrm{Fe}$, and $\mathrm{Ni}$ observations are within the expected abundance range when one takes error bars evaluated from the scatter of Fe and Si below $11000 \mathrm{~K}$ into account. For $\mathrm{Si}$, the trend suggested by the models appears to be present in the data. For Mn our abundances are higher than the results from Behr (2003), which agree well with the predictions. Phosphorus is some five times more overabundant than predicted by the models, whereas magnesium shows small effects, while the models predict about five times larger abundances.

Helium is observed to be more underabundant than expected after $30 \mathrm{Myr}$ (cf. Fig. 7). However, its abundance has a very different time dependence from that of metals. The underabundance of helium is caused by gravitational settling, and this process is as rapid from 10 to $30 \mathrm{Myr}$ as during the first $10 \mathrm{Myr}$. Because helium settles, the settling goes as $\mathrm{e}^{-t / \theta}$, where $\theta$ is the settling time scale. This means that the settling continues exponentially, and if helium is underabundant by a factor of 3 after $30 \mathrm{Myr}$ it should be underabundant by a factor of around 27 after $100 \mathrm{Myr}$, corresponding to a value of 9.56 in Fig. 8 at the end of the $\mathrm{HB}$ evolution. Because it is highly unlikely, however, that most of the stars in Fig. 8 are at the end of their HB evolution, some discrepancy remains.

As may be seen from Fig. 4 of Michaud et al. (2011), abundance anomalies caused by atomic diffusion are not limited to a thin surface phenomenon. However, there are species (e.g. Mg) whose observed anomalies do not agree with the expected ones, suggesting that the model may be missing something. Assuming that the outer $10^{-7} M_{\odot}$ is completely mixed could be an oversimplification, especially since the mixing mechanism is currently unknown. For instance, it might be thermohaline convection (see Sect 5.3 of Michaud et al. 2011). This is a relatively weak convection that might not eliminate all effects of additional diffusion in the atmosphere. For instance, helium is largely neutral in the atmosphere of $\mathrm{HB}$ stars of $11000 \mathrm{~K}$ to $15000 \mathrm{~K}$ so that it has an atomic diffusion coefficient larger than that of ionized metals (by a factor of around 100); helium might then be the most affected atomic species as turbulence weakens. This remains speculative.

\section{Line profile fitting with stratified model spectra}

We also examined how the use of stratified instead of homogeneous model atmospheres to fit our observations influenced the parameters determined from these fits. To allow a direct comparison with results obtained using homogeneous model spectra without metal lines to fit our observations, we calculated a set of synthetic spectra with only $\mathrm{H}$ present (the abundance of all of the other elements were set at -5.00 , while $\mathrm{H}$ was at 12.00 ) while using the relations between temperature, density, and radius from the stratified model atmospheres. Helium lines were not included because the stratification abundances of helium are extremely low. For comparison, we also fitted the observed spectra again (only $\mathrm{H}$ lines) with homogeneous model spectra computed with

\footnotetext{
2 The models around 12000 and $13500 \mathrm{~K}$ were stopped around $10 \mathrm{Myr}$ because of convergence problems, but the trend can be estimated from the surrounding models.
}

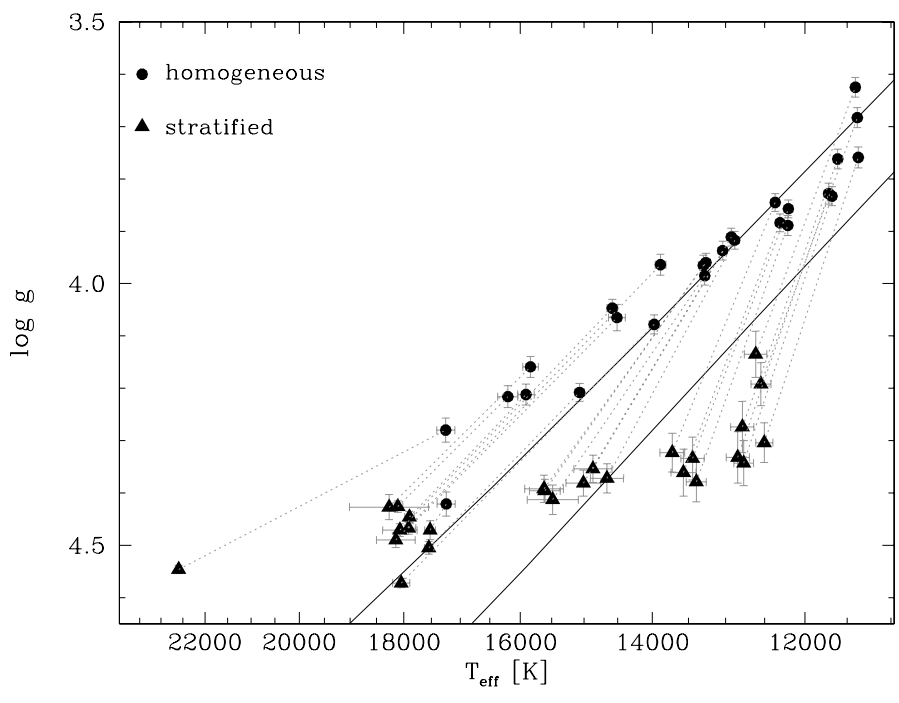

Fig. 10. Atmospheric parameters derived from the line profile fitting for stars hotter than the $u$-jump, using homogeneous model atmospheres $\left([\mathrm{M} / \mathrm{H}]=+0.5, \log \frac{n_{\mathrm{He}}}{n_{\mathrm{H}}}=-3\right.$, filled circles $)$ and stratified model atmospheres (filled triangles). For comparison we also show a canonical zero-age HB and terminal-age HB from Moehler et al. (2003).

$[\mathrm{M} / \mathrm{H}]=+0.5$ and $\log \frac{n_{\mathrm{He}}}{n_{\mathrm{H}}}=-3$. The results are shown in Fig. 10 . The prediction of higher spectroscopic gravities by the models including abundance stratification shown here was also found in Hui-Bon-Hoa et al. (2000) and LeBlanc et al. (2010). The amplitude of the effect might be overestimated by the stratified models because in most cases, they predict stronger abundance anomalies for blue HB stars than observed (see Sect. 6).

In Fig. 10 all stars move to higher temperatures and surface gravities when fitted with model spectra from stratified model atmospheres (as expected from the results of the reverse fitting given in Table 5), but only stars between $14000 \mathrm{~K}$ and $16000 \mathrm{~K}$ move closer to the ZAHB. Cooler stars show too high surface gravities, while hotter ones move parallel to the TAHB. The principal defect of the stratified models is that they generally predict larger abundances than observed. In more realistic models, one would expect that the shifts shown in Fig. 10 be somewhat smaller. The lower temperature stars could then possibly fall between the TAHB and ZAHB in the $\log g-T_{\text {eff }}$ plane, which would give satisfactory results.

As a consistency check we derived masses for the new parameters, adjusting the theoretical $y$-magnitudes of Kurucz (1993) for $[\mathrm{M} / \mathrm{H}]=+0.5$ by -0.3 to account for the stratification effects. This offset was determined by comparing the fluxes of stratified and homogeneous model spectra. The results are shown in Fig. 11. Here the values obtained from stratified model spectra for stars above $14000 \mathrm{~K}$ are now closer to the canonical values than those obtained from homogeneous model spectra. This provides some support to the notion that the too low surface gravities and masses found from analyses with homogeneous model spectra are caused by the mismatch in atmospheric structure between homogeneous and stratified model atmospheres. One should keep in mind, however, that the stratified model atmospheres in general predict much stronger lines than observed, therefore it is currently unclear whether a fully self-consistent solution can be achieved. Similarly to the discussion related to the large shifts found in Fig. 10, the masses predicted in Fig. 11 by more realistic stratified models for stars just above the temperature threshold of the $u$-jump, in which the abundances are less extreme, would be closer to the ZAHB. 


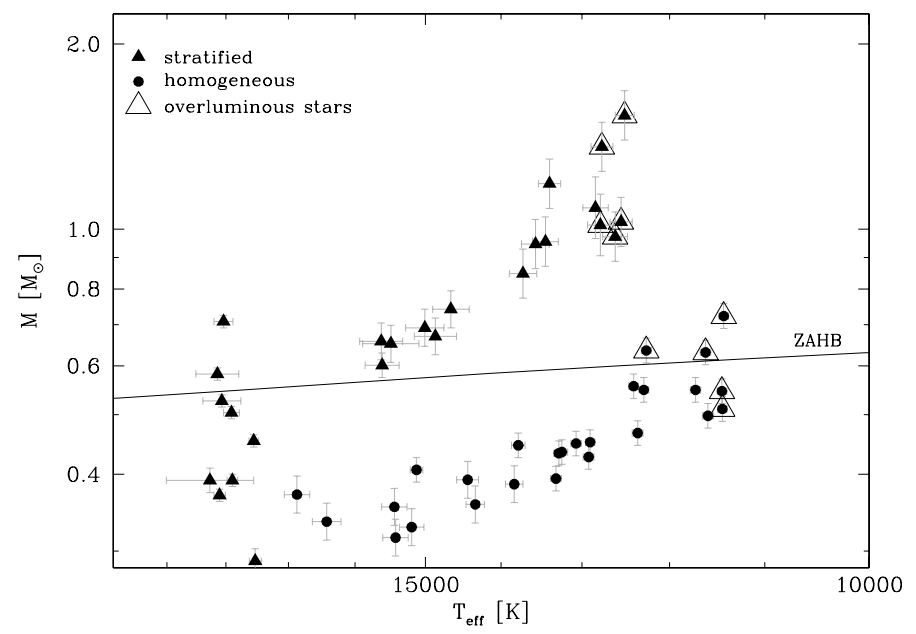

Fig. 11. Masses determined from line profile fits for stars bluer than the Grundahl jump, using homogeneous (filled circles) and stratified (filled triangles) model spectra.

\section{Overluminous stars}

One possible explanation for the overluminous stars mentioned in Sect. 1 is that they have evolved from hotter locations blueward of the $u$-jump and are now near the end of their HB phase, evolving towards the asymptotic giant branch. Because the evolution away from the zero-age HB increases the overall luminosity of an HB star, one would expect such evolved stars to be overluminous in other bandpasses in addition to $u$ compared with stars near the zero-age HB. Figure 12 shows the relation between the effective temperature derived from line profile fits and the $y$-magnitude of the HB stars. Here especially the hot overluminous stars are clearly brighter than the "normal" stars at similar temperatures. To a lesser extent, this is also true for the cool overluminous stars. In Figs. 4 and 5, however, only three of the overluminous stars, namely 100 (hot), 103, and 127 (both cool) are clearly separated from the majority of the stars by a significantly lower surface gravity. It is unclear why the other overluminous stars have higher luminosities, because they are inconspicuous with respect to the majority of the stars in all other parameters (effective temperature, surface gravity, and abundances). However, even three stars evolving off the ZAHB in the temperature range $9700 \mathrm{~K}$ to $11500 \mathrm{~K}$ pose a problem for evolutionary time-scales, as one would expect to find about $100 \mathrm{HB}$ stars close to the ZAHB between about $11000 \mathrm{~K}$ and $14000 \mathrm{~K}$ (corresponding to a range of roughly $0.5-1.05$ in $u-y$ in Fig. 1) for each of the evolved stars, which is clearly not the case.

If the overluminous stars are post-HB stars evolved from a hotter location along the blue tail (as suggested by their higher luminosity), one would expect them to preserve their abundance anomalies during their evolution towards cooler temperatures until dredge-up from the deepening hydrogen convection zone, which causes the surface abundances to return to approximately the normal cluster abundances. However, such stars are expected to have had a low He abundance on the $\mathrm{HB}$ and a low rotation rate, which may cause them to maintain their abundance anomalies to temperatures somewhat lower than the $u$-jump. First, due to the depletion of their surface helium, the overluminous stars just redward of the $u$-jump may have slightly shallower convection zones than other stars of the same effective temperature. However, hydrogen ionization is the main driver of the convection when the effective temperature is lower than

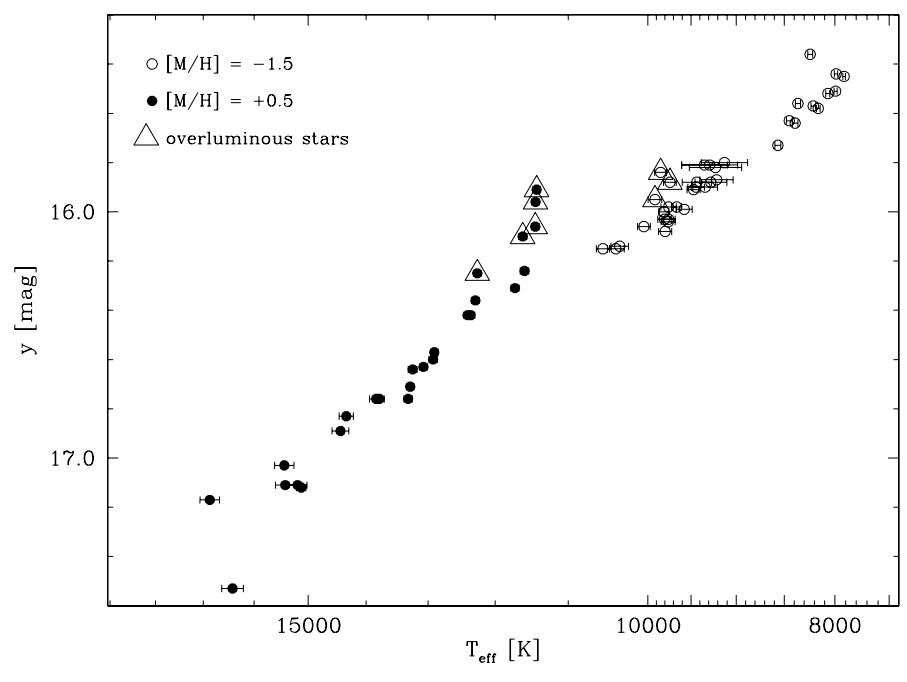

Fig. 12. The $y$-magnitudes of the HB stars compared with the effective temperatures derived from line profile fits.

$10000 \mathrm{~K}$ and it will lead to progressive helium dredge-up so that the difference in convection zone depth could be small below $10000 \mathrm{~K}$. Second, because of the rotation-rate drop in HB stars blueward of the $u$-jump, the overluminous stars would be expected to have low rotation rates if they are evolved from the blue HB. This, in turn, would reduce the meridional flows that inhibit diffusion in stars cooler than the $u$-jump. However, as may be seen from Fig. 4 of Quievy et al. (2009), the limiting rotation rate decreases very rapidly as the effective temperature drops below $10000 \mathrm{~K}$; a star with a $v_{\mathrm{e}}=8 \mathrm{~km} \mathrm{~s}^{-1}$ would show strong effects of diffusion above $12000 \mathrm{~K}$ but would be mixed below $10000 \mathrm{~K}$. The results given in Fig. 8 indeed show that the effects of radiative diffusion disappear between the overluminous stars hotter than the $u$-jump at $11500 \mathrm{~K}$ and the overluminous stars cooler than the $u$-jump at $10000 \mathrm{~K}$. Accordingly, the low He abundance due to diffusion and low rotation apparently does not prevent the effects of diffusion from disappearing rather quickly as a star evolves through the $u$-jump region to cooler temperatures. This result supports the general assumption that the abundances of HB stars redward of the $u$-jump are not affected by diffusion, irrespective of their evolutionary status. The observations are ahead of the stellar evolution models in this case. To set the theory on a stronger base, the evolution calculations with diffusion need to be continued to the end of the $\mathrm{HB}$; but meridional circulation needs to be included as well to verify at which effective temperature the mixing occurs and how this temperature depends on rotation.

\section{Summary and conclusions}

- The atmospheric parameters and masses of the hot HB stars in NGC 288 show a behaviour also seen in other clusters for temperatures between $9000 \mathrm{~K}$ and $14000 \mathrm{~K}$. Outside this temperature range, however, they follow the results found for such stars in $\omega$ Cen.

- The abundances derived from our observations are for most elements within the abundance range expected from evolutionary models that include the effects of atomic diffusion and assume a surface mixed mass of $10^{-7} M_{\odot}$, as determined previously for sdB stars and other clusters. The exceptions are helium, which is more deficient than expected, and phosphorus, which is substantially more abundant than 
predicted. The abundances predicted by stratified model atmospheres, which were not adjusted to observations, are generally significantly more extreme than observed, except for magnesium.

- When the observed spectra were analysed with stratified model spectra, the HB stars were moved to higher temperatures, surface gravities, and masses. Since the equilibrium abundances led to excessive adjustments, more realistic abundance gradients may well lead to models that locate the HB stars between the TAHB and ZAHB (see Sect. 7). Model atmospheres including such improvements are needed to answer this question.

- Five of the eight overluminous stars around the $u$-jump that we observed do not deviate substantially from the other HB stars in the same temperature range in any of the parameters we determined. We are therefore at a loss to explain their brighter luminosities. The remaining three overluminous stars do show lower surface gravities, as would be expected if they evolved away from the HB. However, they pose a substantial problem for evolutionary time-scales, because one would expect to find approximately $100 \mathrm{HB}$ stars close to the ZAHB between about $11000 \mathrm{~K}$ and $14000 \mathrm{~K}$ (corresponding to a range of roughly $0.5-1.05$ in $u-y$ in Fig. 1) for each of the evolved stars, which is clearly not the case.

- All overluminous stars show the same abundance behaviour as the majority of the stars in the respective temperature range. This result supports the general assumption that the abundances of HB stars redward of the $u$-jump are not affected by diffusion, irrespective of their evolutionary status.

Evolution models including diffusion and stratified model atmospheres both predict higher-than-observed abundances for many elements affected by radiative levitation. Evolution models can be adjusted to reproduce the observed abundances by introducing an ad hoc defined mixed zone, which is potentially inconsistent with the observed vertical stratification of at least some elements in the atmosphere, however. For stratified model atmospheres it is currently unclear whether a more limited abundance stratification, which would provide a better description of the observed abundances, would still be able to explain the photometric anomalies around the $u$-jump. Our results show definite promise towards solving the long-standing problem of surface gravity and mass discrepancies for hot HB stars, but much work is still needed to arrive at a self-consistent solution.

Acknowledgements. We thank André Drews for his careful reduction of the data. We are grateful to Christian Moni Bidin for sending his results to allow direct comparisons and for providing a very thorough and helpful referee report.

\section{References}

Behr, B. B. 2003, ApJS, 149, 67

Behr, B. B., Cohen, J. G., McCarthy, J. K., \& Djorgovski, S. G. 1999, ApJ, 517, L135

Behr, B. B., Cohen, J. G., \& McCarthy, J. K. 2000a, ApJ, 531, L37

Behr, B. B., Djorgovski, S. G., Cohen, J. G., et al. 2000b, ApJ, 528, 849
Carretta, E., Gratton, R. G., Clementini, G., \& Fusi Pecci, F. 2000, ApJ, 533, 215 Carretta, E., Bragaglia, A., Gratton, R., D’Orazi, V., \& Lucatello, S. 2009a, A\&A, 508, 695

Carretta, E., Bragaglia, A., Gratton, R., \& Lucatello, S. 2009b, A\&A, 505, 139

Carretta, E., Bragaglia, A., Gratton, R., D’Orazi, V., \& Lucatello, S. 2011, A\&A, $535, \mathrm{~A} 121$

Crocker, D. A., Rood, R. T., \& O’Connell, R. W. 1988, ApJ, 332, 236

Drews, A. 2005, Diploma Thesis, Christian-Albrechts Universität Kiel

Fabbian, D., Recio-Blanco, A., Gratton, R. G., \& Piotto, G. 2005, A\&A, 434, 235

Ferraro, F. R., Paltrinieri, B., Pecci, F. F., et al. 1998, ApJ, 500, 311

Gratton, R. G., Carretta, E., Bragaglia, A., Lucatello, S., \& D’Orazi, V. 2010, A\&A, 517, A81

Grundahl, F., Catelan, M., Landsman, W. B., Stetson, P. B., \& Andersen, M. I. 1999, ApJ, 524, 242

Harris, W. E. 1996, AJ, 112, 1487 (version Dec. 2010)

Horne, K. 1986, PASP, 98, 609

Hui-Bon-Hoa, A., LeBlanc, F., \& Hauschildt, P. H. 2000, ApJ, 535, L43

Khalack, V. R., LeBlanc, F., Bohlender, D., Wade, G. A., \& Behr, B. B. 2007, A\&A, 466, 667

Khalack, V. R., LeBlanc, F., Behr, B. B., Wade, G. A., \& Bohlender, D. 2008, A\&A, 477, 641

Khalack, V., LeBlanc, F., \& Behr, B. B. 2010, MNRAS, 407, 1767

Kurucz, R. L. 1993, ATLAS9 Stellar Atmospheres Program and $2 \mathrm{~km} \mathrm{~s}^{-1}$ grid, CR-ROM No. 13

Lane, R. R., Kiss, L. L., Lewis, G. F., et al. 2010, MNRAS, 406, 2732

LeBlanc, F., Monin, D., Hui-Bon-Hoa, A., \& Hauschildt, P. H. 2009, A\&A, 495, 937

LeBlanc, F., Hui-Bon-Hoa, A., \& Khalack, V. R. 2010, MNRAS, 409, 1606

Michaud, G., Vauclair, G., \& Vauclair, S. 1983, ApJ, 267, 256

Michaud, G., Richer, J., \& Richard, O. 2008, ApJ, 675, 1223

Michaud, G., Richer, J., \& Richard, O. 2011, A\&A, 529, A60

Moehler, S. 2001, PASP, 113, 1162

Moehler, S., Heber, U., \& de Boer, K. S. 1995, A\&A, 294, 65

Moehler, S., Heber, U., \& Rupprecht, G. 1997, A\&A, 319, 109

Moehler, S., Sweigart, A. V., Landsman, W. B., \& Heber, U. 2000, A\&A, 360, 120

Moehler, S., Landsman, W. B., Sweigart, A. V., \& Grundahl, F. 2003, A\&A, 405, 135

Moehler, S., Dreizler, S., Lanz, T., et al. 2011, A\&A, 526, A136

Monelli, M., Milone, A. P., Stetson, P. B., et al. 2013, MNRAS, 431, 2126

Moni Bidin, C., Moehler, S., Piotto, G., Momany, Y., \& Recio-Blanco, A. 2007, A\&A, 474, 505

Moni Bidin, C., Moehler, S., Piotto, G., Momany, Y., \& Recio-Blanco, A. 2009, A\&A, 498, 737

Moni Bidin, C., Villanova, S., Piotto, G., Moehler, S., \& D’Antona, F. 2011, ApJ, 738, L10

Moni Bidin, C., Villanova, S., Piotto, G., et al. 2012, A\&A, 547, A109

Moon, T. T., \& Dworetsky, M. M. 1985, MNRAS, 217, 305

Napiwotzki, R. 1997, A\&A, 322, 256

Napiwotzki, R., Yungelson, L., Nelemans, G., et al. 2004, in Spectroscopically and Spatially Resolving the Components of the Close Binary Stars, eds. R. W. Hilditch, H. Hensberge, \& K. Pavlovski, ASP Conf. Ser., 318, 402

Pace, G., Recio-Blanco, A., Piotto, G., \& Momany, Y. 2006, A\&A, 452, 493

Pasquini, L., Avila, G., Allaert, E., et al. 2000, in Optical and IR Telescope Instrumentation and Detectors, eds. M. Iye, \& A. F. Moorwood, SPIE Conf. Ser., 4008, 129

Peterson, R. C., Rood, R. T., \& Crocker, D. A. 1995, ApJ, 453, 214

Piotto, G., Milone, A. P., Marino, A. F., et al. 2013, ApJ, 775, 15

Quievy, D., Charbonneau, P., Michaud, G., \& Richer, J. 2009, A\&A, 500, 1163

Roh, D.-G., Lee, Y.-W., Joo, S.-J., et al. 2011, ApJ, 733, L45

Salgado, C., Moni Bidin, C., Villanova, S., Geisler, D., \& Catelan, M. 2013, A\&A, 559, A101

Székely, P., Kiss, L. L., Szatmáry, K., et al. 2007, Astron. Nachr., 328, 879

ten Bruggencate, P. 1927, Sternhaufen (Berlin: Springer)

Tonry, J., \& Davis, M. 1979, AJ, 84, 1511

Yong, D., Grundahl, F., Johnson, J. A., \& Asplund, M. 2008, ApJ, 684, 1159

Pages 13 to 14 are available in the electronic edition of the journal at http: //www . aanda.org 
S. Moehler et al.: Hot horizontal-branch stars in NGC 288 - diffusion and stratification effects

Table 1. Target coordinates and photometric data.

\begin{tabular}{|c|c|c|c|c|c|c|}
\hline ID & $\alpha_{2000}$ & $\delta_{2000}$ & $y$ & $b-y$ & $c_{1}$ & $m_{1}$ \\
\hline 52 & $00: 52: 50.77$ & $-26: 38: 02.9$ & $15.357 \pm 0.001$ & $+0.030 \pm 0.003$ & $+1.301 \pm 0.005$ & $+0.131 \pm 0.005$ \\
\hline 55 & $00: 52: 47.43$ & $-26: 33: 14.2$ & $15.318 \pm 0.002$ & $+0.094 \pm 0.002$ & $+1.253 \pm 0.004$ & $+0.122 \pm 0.004$ \\
\hline 60 & $00: 52: 38.59$ & $-26: 37: 05.1$ & $15.328 \pm 0.003$ & $+0.110 \pm 0.004$ & $+1.202 \pm 0.008$ & $+0.118 \pm 0.007$ \\
\hline 61 & $00: 52: 42.80$ & $-26: 36: 39.2$ & $15.312 \pm 0.001$ & $+0.127 \pm 0.002$ & $+1.151 \pm 0.004$ & $+0.121 \pm 0.003$ \\
\hline 63 & $00: 52: 47.08$ & $-26: 35: 25.0$ & $15.345 \pm 0.002$ & $+0.094 \pm 0.003$ & $+1.264 \pm 0.005$ & $+0.119 \pm 0.005$ \\
\hline 70 & $00: 52: 45.40$ & $-26: 35: 21.5$ & $15.438 \pm 0.002$ & $+0.055 \pm 0.003$ & $+1.288 \pm 0.004$ & $+0.133 \pm 0.004$ \\
\hline 72 & $00: 52: 53.92$ & $-26: 38: 45.6$ & $15.421 \pm 0.002$ & $+0.088 \pm 0.004$ & $+1.234 \pm 0.008$ & $+0.125 \pm 0.008$ \\
\hline 74 & $00: 52: 35.47$ & $-26: 34: 24.8$ & $15.453 \pm 0.001$ & $+0.055 \pm 0.002$ & $+1.283 \pm 0.005$ & $+0.133 \pm 0.003$ \\
\hline 79 & $00: 52: 42.68$ & $-26: 34: 50.2$ & $15.507 \pm 0.002$ & $+0.050 \pm 0.003$ & $+1.307 \pm 0.004$ & $+0.135 \pm 0.005$ \\
\hline 81 & $00: 52: 40.77$ & $-26: 33: 47.7$ & $15.569 \pm 0.001$ & $+0.034 \pm 0.002$ & $+1.288 \pm 0.003$ & $+0.137 \pm 0.003$ \\
\hline 83 & 00:52:32.07 & $-26: 35: 46.6$ & $15.560 \pm 0.002$ & $+0.022 \pm 0.002$ & $+1.266 \pm 0.007$ & $+0.139 \pm 0.004$ \\
\hline 86 & $00: 52: 52.51$ & $-26: 34: 29.7$ & $15.582 \pm 0.001$ & $+0.038 \pm 0.002$ & $+1.284 \pm 0.006$ & $+0.136 \pm 0.004$ \\
\hline 88 & $00: 52:$ & $-26: 34: 12.1$ & $15.639 \pm 0.002$ & & $.260 \pm 0.007$ & 0.005 \\
\hline 89 & $00: 52: 3$ & $-26: 36: 35.4$ & $15.518 \pm 0$ & +0.04 & .006 & .003 \\
\hline 90 & $00: 52$ & $-26: 3$ & 15.6 & +0.02 & .011 & 009 \\
\hline 96 & $00: 5$ & $-26: 3$ & .004 & $+0.005 \pm$ & .010 & 007 \\
\hline 99 & 00:52:29.92 & $-26: 36: 07.4$ & $15.799 \pm 0.002$ & $-0.004 \pm 0.003$ & .009 & .007 \\
\hline 100 & $00: 52: 38.68$ & $-26: 35: 58.6$ & $15.959 \pm 0.002$ & $-0.034 \pm 0.002$ & $+0.858 \pm 0.004$ & $+0.112 \pm 0.004$ \\
\hline 101 & $00: 53: 04.16$ & $-26: 38: 29.8$ & $15.911 \pm 0.005$ & $-0.024 \pm 0.009$ & $+0.938 \pm 0.011$ & $+0.116 \pm 0.015$ \\
\hline 102 & $00: 52: 51.47$ & $-26: 36: 26.0$ & $15.815 \pm 0.001$ & $+0.007 \pm 0.002$ & $+1.205 \pm 0.004$ & $+0.128 \pm 0.003$ \\
\hline 103 & $00: 52: 35.45$ & $-26: 39: 11.0$ & $15.840 \pm 0.003$ & $-0.011 \pm 0.004$ & $+1.063 \pm 0.010$ & $+0.115 \pm 0.007$ \\
\hline 106 & $00: 53: 02.55$ & $-26: 35: 32.9$ & $15.822 \pm 0.002$ & $+0.005 \pm 0.003$ & $76 \pm 0.008$ & $+0.127 \pm 0.005$ \\
\hline 107 & $00: 52: 32.39$ & $-26: 36: 30.2$ & $15.810 \pm 0.002$ & $+0.008 \pm 0.002$ & $+1.173 \pm 0.009$ & $+0.126 \pm 0.004$ \\
\hline 111 & 00:53:05.31 & $-26: 32: 45.2$ & $15.982 \pm 0.003$ & $-0.017 \pm 0.004$ & $+1.084 \pm 0.005$ & $+0.136 \pm 0.005$ \\
\hline 113 & $00: 52: 39.48$ & $-26: 36: 45.7$ & $15.866 \pm 0.001$ & $+0.001 \pm 0.002$ & $+1.161 \pm 0.006$ & $+0.125 \pm 0.004$ \\
\hline 114 & $00: 52: 38.68$ & $-26: 37: 49.6$ & $15.905 \pm 0.004$ & $-0.002 \pm 0.006$ & $2 \pm 0.015$ & $26 \pm 0.011$ \\
\hline 115 & $00: 52: 45.24$ & $-26: 37: 55.1$ & $15.881 \pm 0.001$ & $+0.000 \pm 0.002$ & $0 \pm 0.006$ & $5 \pm 0.005$ \\
\hline 118 & $00: 52: 55.50$ & $-26: 35: 08$ & $2 \pm 0.001$ & $5 \pm 0.0$ & $8 \pm 0.008$ & $4 \pm 0.003$ \\
\hline 119 & $00: 5$ & -26 : & 15 & & 0.007 & 0.004 \\
\hline 120 & 00:53:01.85 & $-26: 37: 53.2$ & $15.913 \pm 0.004$ & $0.008 \pm 0.006$ & 007 & .010 \\
\hline 122 & $00: 52: 37.78$ & $-26: 39: 31.6$ & $15.880 \pm 0.002$ & $-0.012 \pm 0.004$ & $+1.094 \pm 0.011$ & $27 \pm 0.009$ \\
\hline 127 & $00: 52: 39.32$ & $-26: 34: 31.6$ & $15.953 \pm 0.002$ & $-0.014 \pm 0.002$ & $+1.047 \pm 0.004$ & $+0.111 \pm 0.004$ \\
\hline 142 & $00: 52: 50.55$ & $-26: 36: 49.8$ & $16.061 \pm 0.002$ & $-0.032 \pm 0.002$ & $+0.876 \pm 0.005$ & $+0.111 \pm 0.004$ \\
\hline 143 & $00: 52: 52.77$ & $-26: 34: 53.0$ & $16.003 \pm 0.002$ & $-0.005 \pm 0.003$ & $+1.084 \pm 0.006$ & $+0.119 \pm 0.006$ \\
\hline 145 & $00: 52: 40.58$ & $-26: 32: 48.6$ & $15.981 \pm 0.002$ & $-0.013 \pm 0.002$ & $+1.108 \pm 0.004$ & $+0.124 \pm 0.003$ \\
\hline 146 & $00: 52: 43.35$ & $-26: 37: 56.4$ & $16.098 \pm 0.002$ & $-0.019 \pm 0.004$ & $+0.878 \pm 0.005$ & $+0.107 \pm 0.006$ \\
\hline 147 & $00: 52: 37.26$ & $-26: 36: 46.9$ & $16.033 \pm 0.002$ & $-0.013 \pm 0.003$ & $+1.054 \pm 0.007$ & $+0.127 \pm 0.004$ \\
\hline 149 & $00: 52: 33.14$ & $-26: 33: 44.6$ & $4 \pm 0.003$ & $-0.020 \pm 0.004$ & $4 \pm 0.009$ & $2 \pm 0.005$ \\
\hline 151 & $00: 52: 48.32$ & $-26: 32: 57.6$ & $16.032 \pm 0.002$ & $-0.016 \pm 0.003$ & $+1.074 \pm 0.004$ & $+0.131 \pm 0.004$ \\
\hline 154 & $00: 52: 54.89$ & $-26: 37: 11.7$ & $16.058 \pm 0.001$ & $-0.007 \pm 0.002$ & $+1.054 \pm 0.007$ & $+0.112 \pm 0.004$ \\
\hline 156 & $00: 52: 50.53$ & $-26: 35: 12.0$ & $16.039 \pm 0.003$ & $-0.004 \pm 0.005$ & $+1.060 \pm 0.007$ & $+0.112 \pm 0.009$ \\
\hline 157 & $00: 52: 53.76$ & $-26: 39: 08.7$ & $15.992 \pm 0.004$ & $-0.013 \pm 0.007$ & $+1.073 \pm 0.011$ & $+0.123 \pm 0.013$ \\
\hline 167 & $00: 52: 46.42$ & $-26: 34: 07.7$ & $16.093 \pm 0.002$ & $-0.009 \pm 0.003$ & $+1.039 \pm 0.006$ & $+0.112 \pm 0.006$ \\
\hline 169 & $00: 52: 46.50$ & $-26: 31: 30.7$ & $16.153 \pm 0.002$ & $-0.023 \pm 0.003$ & $+0.957 \pm 0.006$ & $19 \pm 0.005$ \\
\hline 176 & 00:52:48.70 & $-26: 34: 00.7$ & $16.140 \pm 0.002$ & $-0.012 \pm 0.003$ & $+0.984 \pm 0.006$ & $+0.113 \pm 0.006$ \\
\hline 179 & $00: 52: 48.17$ & $-26: 35: 19.9$ & $16.236 \pm 0.003$ & $-0.032 \pm 0.006$ & $+0.844 \pm 0.009$ & $+0.108 \pm 0.011$ \\
\hline 180 & 00:52:50.91 & $-26: 36: 09.4$ & $16.153 \pm 0.002$ & $-0.014 \pm 0.003$ & $+0.975 \pm 0.004$ & $+0.111 \pm 0.004$ \\
\hline 183 & 00:52:39.91 & $-26: 37: 23.8$ & $16.254 \pm 0.002$ & $-0.025 \pm 0.004$ & $+0.784 \pm 0.011$ & $+0.105 \pm 0.009$ \\
\hline 187 & 00:52:44.71 & $-26: 35: 31.4$ & $16.307 \pm 0.003$ & $-0.033 \pm 0.005$ & $+0.843 \pm 0.008$ & $+0.116 \pm 0.009$ \\
\hline 195 & $00: 52: 27.41$ & $-26: 35: 58.8$ & $16.422 \pm 0.004$ & $-0.050 \pm 0.005$ & $+0.685 \pm 0.008$ & $+0.122 \pm 0.008$ \\
\hline 196 & 00:52:44.29 & $-26: 35: 53.2$ & $16.357 \pm 0.002$ & $-0.031 \pm 0.002$ & $+0.760 \pm 0.004$ & $+0.105 \pm 0.004$ \\
\hline 199 & $00: 52: 55.57$ & $-26: 32: 58.7$ & $16.425 \pm 0.002$ & $-0.042 \pm 0.002$ & $+0.726 \pm 0.005$ & $+0.113 \pm 0.004$ \\
\hline 212 & $00: 52: 59.33$ & $-26: 39: 00.4$ & $16.605 \pm 0.005$ & $-0.068 \pm 0.009$ & $+0.577 \pm 0.013$ & $+0.135 \pm 0.017$ \\
\hline 213 & $00: 52: 42.83$ & $-26: 31: 06.8$ & $16.627 \pm 0.005$ & $-0.062 \pm 0.005$ & $+0.564 \pm 0.008$ & $+0.128 \pm 0.007$ \\
\hline
\end{tabular}


Table 1. continued.

\begin{tabular}{rllllll}
\hline \hline ID & $\alpha_{2000}$ & $\delta_{2000}$ & $y$ & $b-y$ & $c_{1}$ & $m_{1}$ \\
\hline 216 & $00: 52: 54.57$ & $-26: 33: 20.4$ & $16.572 \pm 0.002$ & $-0.051 \pm 0.005$ & $+0.627 \pm 0.011$ & $+0.117 \pm 0.010$ \\
221 & $00: 52: 52.31$ & $-26: 35: 13.7$ & $16.638 \pm 0.002$ & $-0.037 \pm 0.003$ & $+0.584 \pm 0.007$ & $+0.105 \pm 0.006$ \\
228 & $00: 52: 47.36$ & $-26: 37: 52.6$ & $16.761 \pm 0.002$ & $-0.057 \pm 0.004$ & $+0.495 \pm 0.009$ & $+0.116 \pm 0.008$ \\
230 & $00: 52: 24.33$ & $-26: 35: 23.5$ & $16.759 \pm 0.009$ & $-0.059 \pm 0.014$ & $+0.485 \pm 0.029$ & $+0.114 \pm 0.025$ \\
231 & $00: 52: 47.01$ & $-26: 36: 23.0$ & $16.714 \pm 0.003$ & $-0.057 \pm 0.004$ & $+0.552 \pm 0.008$ & $+0.115 \pm 0.006$ \\
240 & $00: 52: 43.69$ & $-26: 35: 01.7$ & $16.761 \pm 0.003$ & $-0.053 \pm 0.005$ & $+0.534 \pm 0.007$ & $+0.113 \pm 0.008$ \\
242 & $00: 52: 45.02$ & $-26: 37: 35.4$ & $16.828 \pm 0.002$ & $-0.064 \pm 0.005$ & $+0.421 \pm 0.007$ & $+0.121 \pm 0.009$ \\
243 & $00: 52: 44.02$ & $-26: 35: 42.2$ & $16.889 \pm 0.003$ & $-0.043 \pm 0.003$ & $+0.445 \pm 0.006$ & $+0.105 \pm 0.005$ \\
275 & $00: 52: 49.39$ & $-26: 35: 53.7$ & $17.030 \pm 0.006$ & $-0.045 \pm 0.009$ & $+0.391 \pm 0.015$ & $+0.098 \pm 0.016$ \\
288 & $00: 52: 49.30$ & $-26: 38: 19.1$ & $17.115 \pm 0.003$ & $-0.058 \pm 0.004$ & $+0.358 \pm 0.006$ & $+0.104 \pm 0.006$ \\
292 & $00: 53: 00.03$ & $-26: 36: 32.9$ & $17.117 \pm 0.003$ & $-0.068 \pm 0.005$ & $+0.406 \pm 0.008$ & $+0.108 \pm 0.008$ \\
300 & $00: 52: 49.11$ & $-26: 35: 35.1$ & $17.106 \pm 0.003$ & $-0.048 \pm 0.007$ & $+0.362 \pm 0.009$ & $+0.100 \pm 0.013$ \\
304 & $00: 52: 48.60$ & $-26: 33: 17.1$ & $17.173 \pm 0.003$ & $-0.054 \pm 0.005$ & $+0.307 \pm 0.011$ & $+0.107 \pm 0.009$ \\
347 & $00: 52: 50.31$ & $-26: 38: 30.0$ & $17.532 \pm 0.003$ & $-0.066 \pm 0.005$ & $+0.275 \pm 0.009$ & $+0.102 \pm 0.009$ \\
\hline
\end{tabular}

Table 2. Observing times, conditions, and setups.

\begin{tabular}{|c|c|c|c|c|c|c|c|}
\hline \multirow[t]{2}{*}{ Date } & \multirow{2}{*}{$\begin{array}{l}\text { Start } \\
\text { [UT] }\end{array}$} & \multirow[t]{2}{*}{ Seeing } & \multirow[t]{2}{*}{ Airmass } & \multicolumn{2}{|c|}{ Moon } & \multirow[t]{2}{*}{ Setup } & \multirow{2}{*}{$\begin{array}{r}\text { Exposure } \\
\text { time }[\mathrm{s}] \\
\end{array}$} \\
\hline & & & & illum. & dist. & & \\
\hline 2003-07-04 & $08: 15: 23$ & 1.. 5 & 1.15 & 0.21 & 146.9 & LR1 & 2520 \\
\hline 2003-07-04 & 09:06:06 & $1 " .1$ & 1.06 & 0.22 & $147^{\circ} .3$ & LR1 & 2520 \\
\hline 2003-07-04 & $09: 49: 14$ & $1 " .1$ & 1.02 & 0.22 & $147^{\circ} .7$ & LR1 & 2000 \\
\hline 2003-07-05* & $07: 11: 48$ & 1.0 & 1.37 & 0.30 & $153^{\circ} .2$ & LR1 & 2520 \\
\hline $2003-07-05^{*}$ & $07: 56: 47$ & 1 1.2 & 1.21 & 0.30 & $153^{\circ} .4$ & LR1 & 1432 \\
\hline 2003-07-07 & 08:04:26 & 0.9 & 1.15 & 0.52 & $149^{\circ} .7$ & LR1 & 2520 \\
\hline 2003-07-07 & $08: 49: 35$ & 0.8 & 1.06 & 0.53 & $149^{\circ} .4$ & LR1 & 2520 \\
\hline 2003-07-07 & 09:33:38 & $0 .{ }^{\prime} 8$ & 1.02 & 0.53 & $149^{\circ} .2$ & LR1 & 2520 \\
\hline 2003-07-07* & 10:16:53 & $0 . ' 8$ & 1.00 & 0.53 & 148.9 & LR1 & 832 \\
\hline 2003-07-08 & 08:23:19 & 0.6 & 1.10 & 0.63 & $140^{\circ} .2$ & LR1 & 2520 \\
\hline 2003-07-08 & 09:09:37 & $0 ! 7$ & 1.03 & 0.64 & $139^{\circ} .8$ & LR1 & 2520 \\
\hline 2003-07-08* & 09:53:34 & 0.98 & 1.01 & 0.64 & $139^{\circ} .4$ & LR1 & 1417 \\
\hline 2003-07-09 & 09:19:31 & 0.7 & 1.02 & 0.75 & $128^{\circ} .2$ & LR1 & 2520 \\
\hline 2003-07-10 & 09:06:35 & $0 .{ }^{\prime} 6$ & 1.03 & 0.84 & $115^{\circ} .9$ & LR3 & 3000 \\
\hline 2003-07-10 & 10:01:51 & $1 . " 1$ & 1.00 & 0.84 & $115^{\circ} 3$ & LR3 & 661 \\
\hline 2003-07-20 & $05: 36: 53$ & 0.7 & 1.59 & 0.59 & $30^{\circ} 1$ & LR3 & 2520 \\
\hline 2003-07-20 & $06: 20: 22$ & 1.0 & 1.40 & 0.59 & $30^{\circ} .3$ & LR3 & 623 \\
\hline 2003-07-21* & $06: 15: 02$ & 0.6 & 1.34 & 0.50 & $38^{\circ} .0$ & LR3 & 2520 \\
\hline 2003-07-21 & 06:58:04 & $0 .{ }^{\prime} 8$ & 1.18 & 0.49 & $38^{\circ} .2$ & LR3 & 2520 \\
\hline 2003-07-21 & 07:47:32 & $1 " .2$ & 1.07 & 0.49 & 38.5 & LR2 & 2520 \\
\hline 2003-07-21 & 08:30:33 & 1.. 5 & 1.02 & 0.49 & 38.7 & LR2 & 2520 \\
\hline 2003-07-21 & 10:17:49 & 1.. 1 & 1.02 & 0.48 & $39^{\circ} .3$ & LR2 & 1736 \\
\hline 2003-07-22 & 05:03:19 & $0 . .4$ & 1.83 & 0.41 & 46.6 & LR1 & 2520 \\
\hline 2003-07-22 & $05: 46: 25$ & 0.4 & 1.47 & 0.40 & $46^{\circ} .9$ & LR1 & 2520 \\
\hline $2003-07-22$ & $06: 37: 10$ & 0.5 & 1.24 & 0.40 & 47.3 & LR2 & 2520 \\
\hline $2003-07-22$ & $07: 22: 53$ & 0.5 & 1.11 & 0.40 & 47.6 & LR2 & 2520 \\
\hline 2003-07-22 & 08:12:00 & 0.5 & 1.04 & 0.40 & 47.9 & LR2 & 2520 \\
\hline $2003-07-22^{*}$ & 10:21:07 & 0.6 & 1.02 & 0.39 & 48.6 & LR2 & 20 \\
\hline 2003-07-27 & $07: 45: 34$ & 0.9 & 1.04 & 0.04 & $101^{\circ} .2$ & LR1 & 2520 \\
\hline $2003-07-28$ & 05:59:11 & 0.5 & 1.29 & 0.01 & $111^{\circ} .3$ & LR2 & 2520 \\
\hline $2003-07-28$ & $06: 49: 13$ & 0.5 & 1.13 & 0.01 & 111.9 & LR1 & 2520 \\
\hline
\end{tabular}

Notes. ${ }^{(*)}$ Not used in final analysis, usually due to $\mathrm{S} / \mathrm{N}$. 